Draft version August 10, 2018

Preprint typeset using $\mathrm{AT}_{\mathrm{E} X} \mathrm{X}$ style emulateapj v. 11/10/09

\title{
PLANET FORMATION IN HIGHLY INCLINED BINARY SYSTEMS I. PLANETESIMALS JUMP INWARDS AND PILE UP
}

\author{
Ji-Wei Xie ${ }^{1,2}$, Matthew J. Payne ${ }^{2}$, Philippe Thébault ${ }^{3}$, Ji-Lin Zhou ${ }^{1}$, Jian Ge ${ }^{2}$, \\ ${ }^{1}$ Department of Astronomy \& Key Laboratory of Modern Astronomy and Astrophysics in Ministry of Education, Nanjing University, \\ 210093, China \\ ${ }^{2}$ Department of Astronomy, University of Florida, Gainesville, FL, 32611-2055, USA and \\ ${ }^{3}$ Observatoire de Paris, Section de Meudon, F-92195 Meudon Principal Cedex, France
}

Draft version August 10, 2018

\begin{abstract}
Most detected planet-bearing binaries are in wide orbits, for which a high inclination, $i_{B}$, between the binary orbital plane and the plane of the planetary disk around the primary is likely to be common. In this paper, we investigate the intermediate stages - from planetesimals to planetary embryos/cores - of planet formation in such highly inclined cases. Our focus is on the effects of gas drag on the planetesimals' orbital evolution, in particular on the evolution of the planetesimals' semimajor axis distribution and their mutual relative velocities. We first demonstrate that a nonevolving axisymmetric disk model is a good approximation for studying the effects of gas drag on a planetesimal in the highly inclined case $\left(30^{\circ}<i_{B}<150^{\circ}\right)$. We then find that gas drag plays a crucial role, and the results can be generally divided into two categories, i.e., the Kozai-on regime and the Kozai-off regime, depending on the specific value of $i_{B}$. For both regimes, a robust outcome over a wide range of parameters is that, planetesimals migrate/jump inwards and pile up, leading to a severely truncated and dense planetesimal disk around the primary. In this compact and dense disk, collision rates are high but relative velocities are low, providing conditions which are favorable for planetesimal growth, and potentially allow for the subsequent formation of planets.
\end{abstract}

Subject headings: methods: numerical — planets and satellites: formation

\section{INTRODUCTION}

Planets in binaries could be common as stars are usually born in binary or multi-stars systems (Duquennov \& Mavor 1991; Mathieu et al. 2000; Duchêne et al. 2007). Although current observations show that the multiplicity-rate of the detected exoplanet host stars is around 17\% (Mugrauer \& Neuhäuser 2009), this fraction should be considered as a lower limit because of noticeable selection effects against binaries in planet searches (Eggenberger 2010). Most of the currently known planet-bearing binary systems are wide S-types (Eggenberger et al. 2004; Desidera \& Barbieri 2007; Haghighipour 2010), meaning the companion star acts as a distant satellite, typically orbiting the inner starplanet system over $100 \mathrm{AU}$ away. Nevertheless, there are currently four systems with smaller separation of $\sim 20$ AU, including the $\gamma$ Cephei (Hatzes et al. 2003), GJ 86, (Queloz et al. 2000), HD 41004 (Zucker et al. 2004), and HD 196885 (Correia et al. 2008; Chauvin et al. 2010). In addition to the planets in circumprimary (S-type) orbits discussed above, planets in circumbinary (P-type) orbits have been found in two systems (Sigurdsson et al. 2003; Lee et al. 2009). While it is possible that the recent detections of numerous short period eclipsing binaries by the Kepler mission (Prša et al. 2011; Slawson et al. 2011) may promote additional such circumbinary detections, their short periods are likely to preclude their use in the characterization of the circumprimary planets on which we concentrate in this investigation.

The properties and diversities of planet-bearing double-star systems provide additional challenges and

xiejiwei@gmail.com constraints for theories of planet formation. Current theories of the core-accretion model of planet formation in single-planet systems (Lissauer 1993; Armitage 2010) envisage formation proceeding via a number of successive stages. In the first step, sub$\mathrm{mm}$ dust grains are converted into 0.1-1000 kmsized planetesimals, the specific mechanism for which may involve (i) some form of grain coagulation (Weidenschilling \& Cuzzi 1993; Weidenschilling 1997; Johansen et al. 2008; Teiser \& Wurm 2009) and/or (ii) some form of fragmentation due to instabilities in the solid sub-component of the protoplanetary gas disk (Safronov 1969; Goldreich \& Ward 1973; Youdin \& Shu 2002; Youdin \& Goodman 2005; Youdin \& Johansen 2007; Cuzzi et al. 2008). Reviews of these topics are given by Blum \& Wurm (2008) and Chiang \& Youdin (2010). In the second stage, mutual (planetesimal-planetesimal) collisions commence and the planetesimals grow via runaway and oligarchic phases (Wetherill \& Stewart 1989; Kokubo \& Ida 1996, 1998; Rafikov 2003, 2004) to form protoplanetary embryos. Depending on the birth-rate and birth size of planetesimals, there could be a transition phase - "snowball" phase - between the first and second stages (Xie et al. 2010b). In the third stage, giant impacts between these embryos commence, leading to the formation of full-size terrestrial planets and gas-giant cores (Chambers \& Wetherill 1998; Levison \& Agnor 2003; Kokubo et al. 2006).

Perturbative effects from stellar companions render the planet formation process in binary systems even more complex than that in single-star systems. For the first stage of planet formation outlined above, it is still unclear whether and/or how the formation of planetesimals 
from dust is affected by the binary star (Pascucci et al. 2008; Zsom et al. 2010); whereas for the third stage, it is thought that planet formation is probably unaffected or even hastened (Barbieri et al. 2002; Quintana 2004; Quintana \& Lissauer 2006; Quintana et al. 2007; Guedes et al. 2008) in a region that extends to the outer limit for orbital stability, i.e., out to $\sim 3-5$ AU for a typical tight binary of semi-major axis $20 \mathrm{AU}$ (Holman \& Wiegert 1999).

However, the intermediate (second) stage of the formation process is much more problematic, as the outcome of planetesimal-planetesimal collisions is highly sensitive to the relative velocities between the bodies during any encounter (Benz \& Asphaug 1999; Stewart \& Leinhardt 2009). The perturbations from a binary companion can excite planetesimal orbits and increase their mutual impact velocity, $\Delta V$, to values that might exceed their escape velocity or even the critical velocity for the onset of eroding collisions (Heppenheimer 1978; Whitmire et al. 1998).

Some earlier investigations (Marzari \& Scholl 2000) suggested that the combination of binary perturbations and gas damping could force a strong pericenter alignment between planetesimal orbits, reducing $\Delta V$. However the work of Thébault et al. (2006) showed that it is only similarly sized objects that experience this reduction, as in general the preferred pericenter alignment is strongly size-dependent, leading to an increasing $\Delta V$ for objects of different sizes. Applying such an analysis to the $\alpha$-Centauri system $\left(a_{B} \sim 18 \mathrm{AU}\right)$, Thébault et al. (2008, 2009) showed that "accretion-friendly" zones exist only for regions within $\sim 0.5-0.75$ AU of an individual star within the binary system. Furthermore, the situation would become much more unfavorable for planetesimal accretion if the eccentricity and precision of the gas disk are considered (Paardekooper et al. 2008; Beaugé et al. 2010). Put simply, the planetesimals-toembryos stage is strongly affected by binary effects for a wide range of parameter space in a circumprimary protoplanetary disk, and it is probably the main obstacle to the formation of planets in close binaries. Some of the deleterious effects of this gas drag could be mitigated if the effects of a dissipating gas disk are taken into account (Xie \& Zhou 2008), but the effect is rather inefficient for small bodies and requires a long timespan $\left(\sim 10^{6}\right.$ years $)$ for the planetesimals to reach a low $\Delta V$ state. One possible solution to this critical problem has been investigated by Paardekooper \& Leinhardt (2010) and Xie et al. (2010b). Both have shown that dust accretion onto planetesimals may provide a safe way for bodies to grow through the problematic 1 to $50 \mathrm{~km}$ size range for which the perturbed environment of the binary can prevent mutual accretion of planetesimals. However, several problems still remain unclear, such as the planetesimal birth rate and the efficiency of dust accretion.

Much of the previously mentioned work had considered only systems in which the binary and planetesimal disk were coplanar, so Xie \& Zhou (2009) went on to consider the effects of small relative inclinations $\left(i_{B}<10^{\circ}\right)$ between the binary orbit and the plane of the protoplanetary disk. Overall, they found that small relative inclinations significantly favor accretion through a reduction in $\Delta V$. Then Xie et al. (2010a) demonstrated that if the disk has a small inclination, the accretion friendly zone can be pushed out to $\sim 1-2$ AU for a system such as $\alpha$-Centauri.

Marzari et al. (2009) investigated cases of much higher inclination, a situation which may be particularly common amongst intermediate separation binaries $(40-100$ $\mathrm{AU})$, where one does not expect there to be significant alignment between disk and binary plane (Hale 1994; Jensen et al. 2004; Monin et al. 2006, 2007). In their investigation they neglected gas drag, arguing that the planetesimal spends the majority of it's orbit outside of the gas disk and hence drag effects are negligible. However, we feel that this argument is specious, as it ignores two facts which are crucial. One is that the planetesimals will cross the gas disk at high inclination, $\hat{i}$, and very high relative velocities, $V_{\text {rel }}$, thus enhancing gas drag, $F_{d}$. This enhancement could be very significant despite the planetesimal spending less of its orbital time in the disk $\left(t_{i n}\right)$. This is because $F_{d}$ is proportional to the square of $\hat{i}$, , while $t_{i n}$ is inversely proportional to the first power of $\hat{i}$. Thus, in total, the planetesimal experiences an increasing momentum change $\left(F_{d} * t_{i n}\right)$ with increasing $\hat{i}$ from gas drag. The other pertinent fact is that planetesimals in sufficiently inclined orbits are likely to experience significant Kozai-driving Kozai 1962) from the binary companion, and hence spend some significant portion of their time with extremely high eccentricities and associated low pericenters. Given some typical power-law density profile for a proto-planetary gas disk (Hayashi 1981; Pringle 1981; Ida \& Lin 2004), this will mean that such planetesimals will be interacting with an extremely dense region of gas at pericenter, and hence will be likely to suffer significant drag even during their brief passage through the gas disk.

Given the demonstrated importance of inclination and gas drag effects in low inclination binary systems (Thébault et al. 2006; Xie \& Zhou 2009), we feel that a full investigation of planetesimal evolution in highly inclined binary systems requires an adequate treatment of gas damping. As such, we perform N-body simulations of planetesimal dynamics in highly inclined binary systems, adding drag forces to simulate the effects of the planetesimals interacting with a gas disk.

We structure our investigation of the planetesimal dynamics in such highly inclined systems as follows: In $\$ 2$ we investigate the effects of different gas disk models (circular and eccentric, precessing and stationary) and justify our choice of the simplest gas disk model. In 3 we look at the behavior of individual particles as they interact with the gas disk and inclined binary companion, using both analytical and numerical methods. Then in $\$ 4$ we investigate the bulk properties of a disk of planetesimals in such a highly inclined system, showing the overall changes in surface density that can take place, and their dependence on parameters such as the binary configuration, the radial size of the planetesimals and the gas disk density. Discussions of the implications for planet formation and the limitations of our approach are made in \$5. Finally, we summarize in 46 .

$\dagger$ Taking the approximation for high $\hat{i}$ cases i.e., $V_{\text {rel }} \sim \hat{i} V_{k}$, where $V_{k}$ denotes the Keplerian velocity, we get $F_{d} \propto \hat{i}^{2}$ from Eqn 6 


\section{MODEL}

Fig[1 is a sketch of the orbital configuration of the binary stars and the circumprimary disk studied in this paper. The companion star acts as an outer perturber on an inclined orbit with mutual inclination of $i_{B}$ around the inner circumprimary disk. Planetesimals are initially embedded in the gas disk and are always treated as test particles, i.e. their growth and mutual gravitational interactions are not included and their motions are affected only by the gravitational forces from the two stars and the effects of the gas disk. The gas disk may have two effects on the motion of planetesimals. One is the gravity of the gas disk, which is ignored in this paper and will be addressed in detail in our forthcoming paper (see the discussion in $\$ \mathbf{5 . 2}$ ). The other effect is the gas drag, which is generally applied to planetesimals whenever they have non-zero relative velocities $\left(V_{\text {rel }}\right)$ to the gas. Throughout this paper, the disk gravity is ignored and we only focus on the effect of gas drag to investigate the planetesimals' motions in binary star systems of different orbital configurations. We use the Mercury code with the BulirschStoer integrator Chambers 1999) for all the simulations except for the one in Fig 13, which uses the Hermite code as in previous papers (Xie \& Zhou 2008, 2009) in order to calculate the relative velocities of planetesimals. As gas drag is dependent on the relative motion between the gas and planetesimals, we must first address the gas motion.

Three possible scenarios can be envisaged for the evolution of a gaseous disk under the perturbations of an inclined companion (Larwood et al. 1996). One possibility is that the gaseous disk begins to warp and it is disrupted by differential precession if the disk is extremely thin. Planetesimals would evolve in a gas-free environment which is the case studied by Marzari et al. (2009). Another possibility is that the gaseous disk remains coherent but quickly relaxes to become aligned to the binary orbital plane if the disk viscosity is very high. Then the situation becomes close to that of the coplanar case or slightly inclined case, which have been well studied previously (Marzari \& Scholl 2000; Thébault et al. 2006, 2008, 2009; Paardekooper et al.|2008; Xie \& Zhou 2008, 2009; Xie et al. 2010a; Beaugé et al. 2010; Paardekooper \& Leinhardt 2010). The third possibility which is relevant for the present paper, is that the gaseous disk maintains a coherent structure and precesses as a rigid body (i.e., $L_{\text {Disk }}$ precesses around $L_{\text {Binary }}$ as seen in Fig (1). In addition to the above considerations, the inner structure of the gaseous disk (flow line) would be significantly modified if the binary orbit is eccentric (Klev 2000; Paardekooper et al. 2008; Kley \& Nelson 2008).

These effects can result in very complicated gas motions in the disk. In order to understand how gas motion affects the gas drag forces on planetesimals, we first describe four gas disk models ( $(2.1)$, then give the general formula for the gas drag force $(\S 2.2)$ and then finally we test the effects of the gas drag resulting from these four gas disk models (\$2.3). Our goal is to obtain a reliable gas drag model for the planetesimals in highly inclined binary systems.
We consider four models for the gas disk.

1. The Circular Gas Disk model (CGD), where the gas is in a circular orbit in the circumprimary midplane. Following Takeuchi \& Lin (2002), in cylindrical coordinates $(r, z)$, we adopt a gas-density distribution of the form

$$
\rho_{g}(r, z)=f_{d} \rho_{g 0}\left(\frac{r}{A U}\right)^{p} \exp \left(-\frac{z^{2}}{2 h_{g}^{2}}\right),
$$

and a gas velocity

$$
V_{g}(r, z)=V_{k, \text { mid }}\left[1+\frac{1}{2}\left(\frac{h_{g}}{r}\right)^{2}\left(p+q+\frac{q}{2} \frac{z^{2}}{h_{g}^{2}}\right)\right]
$$

where $h_{g}(r)=h_{0}(r / \mathrm{AU})^{(q+3) / 2}$ is the scale height of gas disk, $V_{k, \text { mid }}$ is the Keplerian velocity in the midplane, and $f_{d}$ is a dimensionless scaling factor. The subscript " 0 " indicates the value at 1 AU from the disk center-the primary star. In this paper, we take the Minimum Mass Solar Nebula (hereafter MMSN; Hayashi (1981)) as the nominal gas disk, where $f_{d}=1, p=-2.75, q=-0.5$, $\rho_{g 0}=1.4 \times 10^{-9} \mathrm{~g} . \mathrm{cm}^{-3}$, and $h_{0}=4.7 \times 10^{-2}$ AU. We note that the CGD model is a rather crude simplification because it completely ignores the effects of the companion star on the gas disk evolution. However, to a first approximation, the CGD is reasonable and it is also the disk model most-often adopted by previous studies (Scholl et al. 2007; Thébault et al. 2006, 2008, 2009; Xie \& Zhou 2008, 2009).

2. The Elliptical Gas Disk model (EGD) is the same as the CGD model (same density profile and same flow speed), except that the gas is on an elliptical orbit, with an eccentricity of $e_{g}$ and precession rate of $\omega_{g}$. Rigorous values for $e_{g}$ and $\omega_{g}$ require detailed hydro-dynamical modeling on a case-bycase basis, which is beyond the scope of the investigation we wish to perform. Here we follow an alternative strategy and derive simplified analytic expressions for $e_{g}$ and $\omega_{g}$, based on the available hydro-dynamical results from previous studies. Paardekooper et al. (2008) simulated $e_{g}$ and $\omega_{g}$ for two sets of binaries. Of particular interest are the simulations they performed of a $\gamma$ Cepheilike binary system with mass ratio $q_{B}=0.234$, orbital semi-major $a_{B}=20 \mathrm{AU}$, and eccentricity $e_{B}=0.3$. They found that their results for $e_{g}$ and $\omega_{g}$ depended on the flux limiter that they used in their code. For the "minmod" limiter, the disk was "quiet" with low eccentricity $e_{g}<0.02$ and no precession $\left(\omega_{g}=0\right)$. For the "superbee" limiter, the disk is "excited" with $e_{g}$ up to 0.2 and obvious precession $\left(\omega_{g} \neq 0\right)$. Here we adopt the "superbee" results for our EGD model (the "minmod" is close to CGD). Using Fig.7 and Fig.8 of Paardekooper et al. (2008), we estimate

$$
e_{g} \sim \begin{cases}0 & \text { if } r<0.5 \mathrm{AU} \\ 0.12(r-0.5) & \text { if } 0.5 \leq r \leq 2.5 \mathrm{AU} \\ 0.24-0.02(r-2.5) & \text { if } r>2.5 \mathrm{AU},\end{cases}
$$

\subsection{Gas Disk}


and

$$
\omega_{g} \sim 2 \pi / 1500 \mathrm{yr}
$$

(personal communication with Paardekooper reveals that the original data underlying these simulations has been lost due to disk death, hence the need for us to create an approximate reconstruction directly from the published paper).

We note that, in fact, the disk will never reach a steady state (see the Fig.8 of Paardekooper et al. 2008), and thus the above estimation of $e_{g}$ and $\omega_{g}$ should be considered as an averaged approximation. Note also that Eqn 3 and Eqn 4 are valid only for the $\gamma$ Cephei-like case, a more generic expression for $e_{g}$ and $\omega_{g}$ which includes the dependency on the binary configuration would require more simulation results based on hydro-dynamical modeling.

3. The Circular Gas Disk + Nodal Precession model $(\mathrm{CGD}+\mathrm{N})$, has the same inner structure as the CGD model, but adds in the precession of the ascending node of the disk plane relative to the binary orbital plane. Nodal precession occurs when the disk plane has a considerable tilted angle $\left(i_{B}\right)$ relative to the binary orbital plane. Larwood et al. (1996) found that, if the Mach number of the disk is not too large ( $M a<30$ roughly), then the disk can maintains its structure and undergoes a near rigid body precession at a rate given by

$$
\Omega_{g}=-\frac{15 M_{B} R_{d}^{3}}{32 M_{A} a_{B}^{3}} \cos i_{B} \Omega_{k},
$$

where $M_{A}$ and $M_{B}$ are the masses of the primary and secondary stars, $\Omega_{k}$ is the local Keplerian rotation rate and $R_{d}$ is the radial size of the truncated disk. For the $\gamma$ Cephei like case, we set $R_{d}=4$ AU according to Artymowicz \& Lubow (1994) and Pichardo et al. (2005).

4. The Eccentric Gas Disk + Nodal Precession model $(\mathrm{EGD}+\mathrm{N})$, has the same inner structure as the EGD model and the same nodal precession as the $\mathrm{CGD}+\mathrm{N}$ model.

From physical considerations, EGD $+\mathrm{N}$ is the most realistic disk model of the four. However, as we will see in $\oint 2.3$. the four disk models all result in similar gas drag effects in highly inclined cases.

\subsection{Gas Drag}

A spherical particle of radius $R_{p}$, moving through a gas with a relative velocity $V_{r e l}$, experiences an aerodynamic drag force $F_{d}$ given by (e.g. Armitage 2010)

$$
\mathbf{F}_{\mathbf{d}}=-\frac{C_{D}}{2} \pi \rho_{g} R_{p}^{2} V_{\text {rel }} \mathbf{V}_{\text {rel }}
$$

where $\rho_{g}$ is the gas density and $C_{D}$ is the drag coefficient. The form of the drag coefficient depends upon the size of the particle compared to the mean free path $\lambda$ of molecules in the gas $\left(\lambda \sim 0.27(r / A U)^{-11 / 4} \mathrm{~cm}\right.$ for the MMSN). If $R_{p}<9 / 4 \lambda$, then

$$
C_{D}=\frac{8}{3}\left(\frac{8}{\pi}\right)^{1 / 2} \frac{C_{S}}{V_{r e l}}
$$

where $C_{S} \sim \Omega_{k} h_{g}$ is the local sound speed. This is called the Epstein regime of drag. For larger particles the Stokes drag law is valid, and the drag coefficient can be expressed as a piecewise function

$$
C_{D}= \begin{cases}24 R e^{-1} & \text { if } \quad R e<1 \\ 24 R e^{-0.6} & \text { if } \quad 1 \leq R e \leq 800 \\ 0.44 & \text { if } R e>800\end{cases}
$$

Here $R e=2 R_{p} \rho_{g} V_{\text {rel }} / \mu$ is the Reynolds number, and $\mu=(8 / \pi)^{1 / 2} \rho_{g} C_{s} \lambda / 3$ is the kinematic viscosity of the gas.

\subsection{Model Test and Comparison}

All the tests and comparisons are conducted in the $\gamma$ Cephei-like case as in Paardekooper et al. (2008) with mass ratio $q_{B}=0.234$, orbital semimajor $a_{B}=20 \mathrm{AU}$, and eccentricity $e_{B}=0.3$. Although such a binary configuration is too tight to be relevant for the highly inclined case (usually $a_{B}>30-40$ AU according to Hale (1994)), we still choose it as our test case because hydrodynamic results are currently available only for such close binaries (Paardekooper et al. 2008). In the following, we consider two sets of tests or comparisons.

- We first test the EGD model through comparison (see Fig 2) to the hydro-dynamical simulations of Paardekooper et al. (2008). In our simulation with the EGD model, 100 particles with $R_{p}=1$ and $R_{p}=5 \mathrm{~km}$ respectively were initially set in circular orbits in the coplanar plane with semi-major axes from 0.5 to 5 AU. As can be seen in Fig 2 the EGD model can roughly recover the basis features of Paardekooper's results, such as (1) the shape of the eccentricity profile of planetesimals, and (2) the trend that smaller particles $\left(R_{p}=1 \mathrm{~km}\right)$ behavior more closely matches that of the gas in the inner regions of the disk (because gas drag is more efficient for smaller size particles and denser gas density). Therefore, we believe that the EGD model, though rather simplified, provides a reasonable means to include the basic effects of gas-disk eccentricity induced by the companion's perturbations.

- We then compare the results of the four gas disk models (see Fig 3). We set three planetesimals with radial size $R_{p}=5 \mathrm{~km}$, and with circular orbits starting at $3 \mathrm{AU}$ (i.e., the initial semimajor axis $\left.a_{p 0}=3 \mathrm{AU}\right)$ in the gas disk plane. We consider a near-coplanar case $\left(i_{B}=10^{\circ}\right)$, an intermediately inclined case $\left(i_{B}=30^{\circ}\right)$ and a highly inclined case $\left(i_{B}=60^{\circ}\right)$ for which the Kozai effect should be apparent. As can be seen in Fig 3. only for the near-coplanar case does the gas eccentricity have any significant effect on the evolution of the planetesimal semi-major damping. That is because the gas drag force depends mainly on the planetesimal's orbital inclination with respect to the gas disk plane in significantly inclined binary cases (see also in Fig 5 and Fig 6 ). In addition, we see that the nodal precession of the gas disk has little effect for all the cases. This is well understood as the planetesimal node always undergoes nodal precession independent of whether the nodal precession 
of the gas disk occurs or not. A detailed investigation of the planetesimal dynamics, including the reason for large inward jumps evident in Fig 3 is provided in $\S 3$.

In summary, through the above comparisons, we see that the most commonly adopted model, i.e., the CGD model, is only a rather crude simplification in the near coplanar cases $\left(i_{B}<10^{\circ}\right)$, but it is a good approximation in highly inclined cases $\left(i_{B} \geq 30\right)$. This conclusion, although obtained from a test on a specific binary with a $\gamma$ Cephei-like configuration, should apply to binaries with any separation since there is not any significant separation dependence as seen from the above test and comparison. The EGD $+\mathrm{N}$ model, though more realistic, is currently available only for close binaries with separation of 10-20 AU, while the CGD model can readily be applied to any case. Therefore, given our interests in this paper in modeling highly inclined binaries with separation of $>30-40$ AU, hereafter we adopt the CGD as our standard gas-disk model, and focus on it as we try to understand the planetesimal behavior in binary systems with different configurations $\left(a_{B}, e_{B}, i_{B}\right)$ and mass ratios $\left(q_{B}\right)$.

\section{DYNAMICS OF INDIVIDUAL PLANETESIMALS: INWARD JUMPING}

\subsection{Different Regimes of Semimajor Axis Damping}

Before presenting detailed numerical results, we present in this chapter analytical considerations in order to understand the different regimes in which gas drag can operate on planetesimals in a circumprimary disk.

The main effect of gas friction is to force an inward drift of planetesimals. According to Adachi et al. (1976), the average migration rate (or the semi-major axis damping rate) follows the approximate relation

$$
\begin{aligned}
\frac{\tau_{0}}{a} \frac{\widehat{d a}}{d t} \sim & -2\left(\frac{5}{8} \hat{e}^{2}+\frac{1}{2} \hat{i}^{2}+\eta^{2}\right)^{1 / 2} \\
& \times\left(\eta+\frac{17}{16} \hat{e}^{2}+\frac{1}{8} \hat{i}^{2}\right),
\end{aligned}
$$

where $a, e, i$ are the orbital semimajor axis, eccentricity and inclination (relative to the disk plane rather than the binary orbit plane) of the planetesimal, the hat symbol (e.g. $\hat{e}$ and $\hat{i}$ ) denotes the average value of that variable taken over at least one orbital period, $\eta$ denotes the deviation of the gas rotation from the Keplerian velocity, and $\tau_{0}$ is a characteristic timescale depending on the gas density and planetesimal radial size. For a gas disk scaled in proportion to the MMSN, we have $\eta \sim 2 \times 10^{-3}$ and $\tau_{0}$ can be written as

$$
\tau_{0} \sim 24 f_{d}\left(\frac{\hat{i}}{h_{0}}\right)\left(\frac{R_{p}}{\mathrm{~km}}\right)\left(\frac{a}{\mathrm{AU}}\right)^{3}\left(\frac{M_{A}}{M_{\odot}}\right)^{-1 / 2} \mathrm{yr},
$$

where $f_{d}$ is the scaling number of the disk density compared to MMSN, and the factor $\left(\hat{i} / h_{0}\right)$ accounts for the decrease in the gas density in the vertical direction.

Eq. 9] indicates that the migration rate has contributions from three sources. One is $\eta$, which accounts for the fact that the pressure supported gas disk departs from purely Keplerian dynamics and only depends on the properties of the gas disk. The other two are the planetesimal orbital eccentricity $(\hat{e})$ and inclination $(\hat{i})$, which can be excited via various mechanisms by the companion star, and measure the angle between planetesimal orbits and gas streamlines. If, for example, $\hat{i} \gg \hat{e}$ and $\eta$, then Eq9 gives $d a / d t \propto \hat{i}^{2}$; planetesimals undergo a much faster inward migration with increasing $\hat{i}$. This first order estimate indicates that highly inclined planetesimals never evolve in a manner close to the gas-free case, despite the fact that they spend more than $80-90 \%$ of their orbital time in an essentially gas-free environment (Marzari et al. 2009).

Fig 4 is a schematic diagram showing which source or mechanism dominates the inward migration. Three main regions, corresponding to different regimes for gas drag, can basically be distinguished: (1) region $\mathrm{S}$, where planetesimal migration is close to that in an unperturbed, single star case, (2) region B where planetesimal migration is dominated by the binary perturbations, and (3) region $\mathrm{SB}$, a transition region between $\mathrm{B}$ and $\mathrm{S}$. In addition, region $\mathrm{B}$ can be divided into three subregions corresponding to three different mechanisms which can excite the $\hat{e}$ and/or $\hat{i}$. The precise definitions of all these regimes is as follows:

- Region S: where $\frac{5}{8} \hat{e}^{2}<\eta^{2}$ and $\frac{1}{2} \hat{i}^{2}<\eta^{2}$. For this low dynamical excitation case, according to Eq9. the migration rate is dominated by $\eta$. For MMSNlike gas disks, Region S corresponds to planetesimals that are very close to the primary as compared to the distance of the companion, i.e. $a / a_{B} \ll 1$. In this case, $\hat{e}$ and $\hat{i}$ remain very small and planetesimal orbits are close to what they would be in an unperturbed single-star case. Note here that the inward migration rate $\left(\mathrm{Eqn}[9] \widehat{d a / d t} \propto \eta^{2} a / \tau_{0} \propto\right.$ $a^{-1}$, meaning that the inward migration is accelerated in region $\mathrm{S}$.

- Region B: $\hat{e}$ and/or $\hat{i}$ are significantly pumped up by the companion's perturbations and thus dominate the damping rate of planetesimal semimajor axis. Depending on the specific mechanism that pumps up $\hat{e}$ and/or $\hat{i}$, region $\mathrm{B}$ is divided into three subregions:

- subregion B1: where $\hat{e}$ and/or $\hat{i}$ are excited by secular perturbations, but Kozai effects are absent, i.e., $i_{B}<39.2^{\circ}$. If we assume the average values of $\hat{e}$ and $\hat{i}$ approximate their forced values $e_{f}\left(e_{f}<\sim 0.1\right.$, see the Appendix $)$ and $i_{f}\left(i_{f} \sim i_{B}<39.2^{\circ}\right)$, then region B1 corresponds to the middle part of Fig 4 .

- subregion B2, the Kozai regime, i.e., $\hat{i} \sim i_{B}>$ $39.2^{\circ}$. In such case, both $\hat{e}$ and $\hat{i}$ can be highly excited via the Kozai effect.

- subregion B3, where $\hat{e}$ is excited and it is independent of $\hat{i}$. This region may correspond to a mean motion resonance scenario. An example can be found in Ida \& Lin (1996), which shows the combination of gas drag and Jupiter's mean motion resonances can affect the structure of the asteroid belt. 
- Intermediate region SB: where $\frac{5}{8} \hat{e}^{2}>\eta^{2}$ and $\frac{1}{2} \hat{i}^{2}>$ $\eta^{2}$ but $\frac{17}{16} \hat{e}^{2}<\eta$ and $\frac{1}{8} \hat{i}^{2}<\eta$. Region SB corresponds to the transition case, where $\hat{e}$ and $\hat{i}$ are only mildly excited, meaning that the migration rate is determined by the combination of $\eta, \hat{e}$ and/or $\hat{i}$.

The dashed line in Fig प i.e., $\frac{17}{16} \hat{e}^{2}=\frac{1}{8} \hat{i}^{2}$, divides the entire plot into two parts: In the upper-left $\hat{e}$ dominates the damping of semi-major axis, while in the bottomright of the plot $\hat{i}$ contributes more than $\hat{e}$. We define the boundary between regions B1 and B3 as being $\hat{e}=0.1$, since the maximum forced eccentricity $\left(e_{f}\right)$ for a typical binary system is $\sim 0.1$ (see the Appendix for detail).

Note that Fig 4 is based on Eq 9 which is derived from the most simplified gas drag model, i.e. the circular gas disk model (CGD). According to the test in $\S 2.3$ (see also in Fig (3), the CGD model is a good approximation only for relative large $\hat{i}$. Therefore, hereafter we focus only on these large $\hat{i}$ (or $i_{B}$ ) cases, and simply classify them into two categories: Kozai-off (which corresponds to the right-hand section of the B1 region) and Kozai-on (the whole of the B2 region). We don't explore region B3 in the present paper, as it is likely only to be relevant to some isolated (i.e., in resonance) or unstable regions (i.e., close to the companion star) of the protoplanetary disk.

\subsection{Kozai-Off Regime}

In the following sections, we turn to numerical simulations using the circular gas disk model (CGD) which was tested and justified in $\S 2$.

We first define our standard system parameters upon which we base most of our variations. We take $M_{A}=$ $2 M_{B}=M_{\odot}, a_{B}=50 \mathrm{AU}, e_{B}=0.3, i_{B}=30^{\circ}$, $f_{d}=1.0$, and $R_{p}=5 \mathrm{~km}$. The mass ratio and eccentricity are chosen as the typical values for known binaries (Duquennoy \& Mavor 1991). The separation is chosen as it is wide enough to be relevant for large inclination and tight enough to have the binary companion cause considerable perturbations in the $0.1-10 \mathrm{AU}$ region, where the core-accretion model of planet formaiton prefers. $i_{B}=30^{\circ}$ is chosen as it is large enough for the validity of CGD model as tested in $\S 2.3$, but not so large as to induce the Kozai effect. Such a binary configuration leads to a stable orbit limit of $\sim 10 \mathrm{AU}$ around the primary star (Holman \& Wiegert 1999).

This high $\hat{i}$ case corresponds to the right-hand side of the B1 region in Fig 4 Fig 5 presents the result of a numerical integration showing the typical behavior of a planetesimal orbit in this regime (see also in the middle panel of Fig 3 but note different binary parameters). As expected from linear secular perturbation theory, $\hat{e}$ and $\hat{i}$ undergo oscillations with a period equal to the secular perturbation time scale, i.e. $t_{s p}$. The value of $t_{s p}$ derived from Fig 5 is in good agreement with the first order approximation derived by Thébault et al. (2006):

$$
\begin{array}{r}
t_{s p} \sim \frac{4}{3}\left(\frac{a}{\mathrm{AU}}\right)^{-3 / 2}\left(\frac{a_{B}}{\mathrm{AU}}\right)^{3}\left(1-e_{B}^{2}\right)^{3 / 2} \\
\left(\frac{M_{A}}{M_{\odot}}\right)^{1 / 2}\left(\frac{M_{B}}{M_{\odot}}\right)^{-1} \mathrm{yr},
\end{array}
$$

where $M_{A}, M_{B}$, and $M_{\odot}$ are the masses of the primary, secondary and the Sun respectively. As the relative velocity $V_{\text {rel }}$ between the planetesimal and gas can be approximately estimated as (see Eqn 4.20 of Adachi et al. $(1976))$

$$
V_{r e l}^{2} \sim V_{k}^{2}\left(\frac{5}{8} \hat{e}^{2}+\frac{1}{2} \hat{i}^{2}+\eta^{2}\right),
$$

this means that in the regime where $\hat{i} \gg \hat{e}($ and $\eta), V_{\text {rel }}$ and the gas drag force $F_{d}$ (Eqn $\left.\sqrt[6]{ }\right)$ will also follow the same periodic oscillation as $\hat{i}$. This oscillation of gas drag damping causes the planetesimal semimajor axis to damp step-by-step as seen in the bottom panel of Fig.5.

In addition, as can be seen in Fig 5 the oscillation amplitudes of $\hat{i}$ and $\hat{e}$ are under-damping because of gas drag. According to Adachi et al. (1976), the damping of $\hat{i}$ and $\hat{e}$ follows,

$$
\frac{\tau_{0}}{\hat{e}} \frac{\widehat{d e}}{d t} \sim 2 \frac{\tau_{0}}{\hat{i}} \frac{\widehat{d i}}{d t} \sim-\left(\frac{5}{8} \hat{e}^{2}+\frac{1}{2} \hat{i}^{2}+\eta^{2}\right)^{1 / 2} .
$$

In the present case, where $\hat{i} \gg \hat{e}($ and $\eta$ ), the timescale of $\hat{i}$ damping can thus be approximated by

$$
t_{d p} \sim \frac{\hat{i}}{\widehat{d i / d t}} \sim 2^{3 / 2} \frac{\tau_{0}}{\hat{i}} .
$$

Substituting Eq10 into Eq14, $t_{d p}$ can then be rewritten as

$$
t_{d p} \sim 1444 f_{d}^{-1}\left(\frac{R_{p}}{\mathrm{~km}}\right)\left(\frac{a}{\mathrm{AU}}\right)^{3}\left(\frac{M_{A}}{\mathrm{M} \odot}\right)^{-1 / 2} \quad \mathrm{yr}
$$

Depending on whether the secular perturbation or the gas drag damping dominates, Fig 5 can be divided into two parts with the boundary between these two regions being roughly set by the last turn-over point of the migration curves: E.g. for the red-curve in Fig $[5$ the coordinates of the critical time and semi-major axis at which this occurs is $t_{c} \sim 5.75 \times 10^{5}$ yr and $a_{c} \sim 1.5$ AU for $a_{p 0}=7 \mathrm{AU}$. For times earlier than $t_{c}$ or semi-major axes beyond $a_{c}, \hat{i}$ is periodically excited, and thus the planetesimal periodically undergoes a step-wise inward migration with the steep drops in semi-major axis corresponding to the peaks in $\hat{i}$. For times greater than $t_{c}$ or semi-major axes inside $a_{c}, \hat{i}$ is smoothly damped to a low value, thus the planetesimal migrates inward smoothly and slowly.

We can also estimate $a_{c}$ by equating $t_{s p}$ and $t_{d p}(\mathrm{Eq} 11$ and Eq[15), which gives a "zeroth-order" estimation of $a_{c 0}$ as,

$$
\begin{array}{r}
a_{c 0} \sim 0.9 \times f_{d}^{2 / 9}\left(\frac{a_{B}}{10 \mathrm{AU}}\right)^{2 / 3}\left(1-e_{B}^{2}\right)^{1 / 3} \\
\left(\frac{R_{p}}{\mathrm{~km}}\right)^{-2 / 9}\left(\frac{M_{A}}{M_{B}}\right)^{2 / 9} \mathrm{AU} .
\end{array}
$$

We call $a_{c 0}$ "zeroth-order" because this timescale analysis gives the correct dependency of $a_{c}$ on the various parameters (for example, $a_{c}$ is independent of $a_{p 0}$ in Fig (5), but not the correct absolute coefficient. Through many sets of simulations, such as Fig 5 but with different stellar mass ratios $\left(M_{A} / M_{B}=0.2,0.5,1.0\right)$, binary semimajor 
axies $\left(a_{B}=10,20,50,100,200 \mathrm{AU}\right)$, binary eccentricities $\left(e_{B}=0,0.3,0.6\right)$, binary inclinations $\left(i_{B}=20^{\circ}, 30^{\circ}, 39^{\circ}\right.$ and $\left.150^{\circ}\right)$, gas disk densites $\left(f_{d}=0.05,0.5,1\right)$, and planetesimal radial sizes $\left(R_{p}=1,5,100 \mathrm{~km}\right)$, we found similar results for both the prograde and retrograde cases (see the bottom panel of Fig $[5$ and the empirically measured form of $a_{c}$ is just systematically smaller by $\sim 33 \%$ than $a_{c 0}$, i.e.,

$$
\begin{aligned}
a_{c} \sim & \frac{2}{3} a_{c 0} \\
\sim & 0.6 \times f_{d}^{2 / 9}\left(\frac{a_{B}}{10 \mathrm{AU}}\right)^{2 / 3}\left(1-e_{B}^{2}\right)^{1 / 3} \\
& \left(\frac{R_{p}}{\mathrm{~km}}\right)^{-2 / 9}\left(\frac{M_{A}}{M_{B}}\right)^{2 / 9} \mathrm{AU} .
\end{aligned}
$$

We note that $t_{d p}$ is larger by just about one order of magnitude than $t_{s p}$ at $a_{c}$.

The physical meaning of $a_{c}$ is that it indicates the turnover point where the planetesimal semi-major axis transits from the excited (usually fast) damping regime to the quiet (usually slow) damping regime (also corresponding to the boundary between the region B1 and SB). The importance of $a_{c}$ lies in the fact that it determines the location in the proto-planetary disk where planetesimals will pile up (see $\S 4$ for details).

\subsection{Kozai-On regime}

The standard setup for this case (corresponding to the $\mathrm{B} 2$ region in Fig (4) is chosen as similar to that in $\$ 3.2$, but with $i_{B}=60^{\circ}$ instead. As $i_{B}$ is larger than the critical value $39.2^{\circ} \mathrm{V}(\mathrm{R})$, the Kozai effect is turned on (Kozai 1962), and $\hat{e}$ can be excited to a degree which is comparable with $\hat{i}$. In such a case, both $\hat{e}$ and $\hat{i}$ have major contributions to the planetesimal migration rate (see Eqn9).

The most interesting result is that large eccentricity oscillations can lead to very fast inward migration of the planetesimals towards the inner disk. As can be seen in Fig 6 a planetesimal starting at $a_{p 0}=3 \mathrm{AU}$ spirals down to $1 \mathrm{AU}$ in $\sim 10^{4}$ years. For the $a_{p 0}=5 \mathrm{AU}$ case, the planetesimal starts closer to the companion star and is subjected to much stronger Kozai perturbations. Eccentricities can quickly reach values as high as 0.7 that places the planetesimal pericenter in inner gas rich regions where it will in addition reach very high $V_{\text {rel }}$ values. This leads to a very fast, almost "jump" -wise inward migration from $5 \mathrm{AU}$ to $\sim 0.35 \mathrm{AU}$. For the $a_{p 0}=8 \mathrm{AU}$ case, the eccentricity also reaches 0.7 but, due to the larger initial semi-major axis, the planetesimal cannot directly reach very dense inner regions and thus first undergoes several small jumps, until finally making a bigger jump to $\sim 1$ AU.

All the inward jumps occur when $\hat{e}$ and/or $\hat{i}$ is excited, leading to a surge in the experienced gas drag force $\left(F_{d}\right)$. Although the $\hat{e}$ contribution to $F_{d}$ is significant, it seems that $\hat{i}$ is still the major component of $F_{d}$. Note $\hat{i}$ is with respect to the disk plane rather than to the binary orbital plane, and thus the periodic variation of $\hat{i}$ shown in Fig 6 is caused by the Nodal precession of the planetesimal orbit with a period of two times that of the Kozai cycle period.
For all cases, once the planetesimals complete their jump, they will approximately stabilize at that semimajor axis, with very small residual $\hat{e}$ and $\hat{i}$ and thus only very slow inward migration. They no longer suffer from the Kozai effects because they have jumped into an inner region where the gas is so dense that gas drag damping dominates over Kozai excitation. Unlike the Kozai-off case, the turn-over semi-major axis, where the planetesimal stops jumping, is no longer independent of $a_{p 0}$ and not equal to $a_{c}$ (Eqn,17). Fig.7 shows how it varies with $a_{p 0}$ in the Kozai-on case.

- If $a_{p 0} \lesssim 1.5 \mathrm{AU}$, the turnover semi-major axis is around $a_{p 0}$, which means that no "inwardjumping" occurs to the planetesimals in the very inner region; Kozai effects is fully turned off because such dense gas and hence strong gas drag damping dominates over Kozai excitation.

- If $1.5 \lesssim a_{p 0} \lesssim 8 \mathrm{AU}$, the turn-over semi-major axis follows a characteristic oscillatory pattern, whose amplitude varies between a lower limit at $a_{c \text { (low })} \sim$ $0.3 \mathrm{AU}$ and an upper limit at $a_{c} \sim 1.5 \mathrm{AU}$ roughly;

- If $a_{p 0} \gtrsim 8 \mathrm{AU}$, the planetesimal is initially located close to the boundary of the stable orbit $(\sim 10 \mathrm{AU})$, thus the results become chaotic. However most, if not all, of the turn-over points remain confined within the range between $a_{c(l o w)}$ and $a_{c}$, i.e., 0.3$1.5 \mathrm{AU}$.

In Fig 8, we study the dependence of the lower limit of the turn-over semi-major axis $\left(a_{c(l o w)}\right)$ on $a_{B}$ and $i_{B}$. Empirically we found that

$$
a_{c(\text { low })} \sim\left\{\begin{array}{lll}
0.50 a_{c} & \text { if } & i_{B}=50^{\circ} \\
0.20 a_{c} & \text { if } & i_{B}=60^{\circ} \\
0.10 a_{c} & \text { if } & i_{B}=70^{\circ}
\end{array}\right.
$$

where $a_{c}$ is given by Eqn 17. For even larger $i_{B}\left(70^{\circ}-\right.$ $\left.90^{\circ}\right)$, the $a_{c(l o w)}$ turns to be larger. The $a_{c(l o w)}$ for prograde and retrograde cases are symmetrical about $i_{B}=90^{\circ}$. The combination of $a_{c(l o w)}$ and $a_{c}$ determines a region for the Kozai-On case, into which the planetesimals are likely to jump and pile up.

\section{BEHAVIOR OF A SWARM OF PLANETESIMALS: PILING UP}

\subsection{Initial Set-up}

In $\oint[3$, we focused solely on the dynamics of individual planetesimals. In this section, we investigate the behavior of a swarm of planetesimals, focusing on the evolution of the planetesimal disk's surface density $\Sigma$, under the coupled effects of gas drag and binary perturbations.

In each simulation, all planetesimals are initially set onto a near-coplanar circlular orbit around the primary star with orbital eccentricity and inclination (relative to the gas disk plane) $0 \leq e=2 i \leq 10^{-4}$. All other angular elements are randomly distributed. The planetesimals' semimajor axes are uniformly distributed in a range ,i.e., $a_{\text {in }}<a<a_{\text {out }}$ with the same radial size $\left(R_{p}\right)$. We set $a_{i n}=0.05 \mathrm{AU}$, within which planetesimals are removed from the simulation. $a_{\text {out }}$ is set as the boundary of the stable orbit according to Holman \& Wiegert (1999). In order to obtain reliable statistical results, we initially put 
$N_{p 0}=100$ planetesimals in every semimajor axis bin of 1 AU width. By monitoring the planetesimal number $\left(N_{p}\right)$ in each bin, we can estimate the change of planetesimal surface density, i.e., $\Sigma / \Sigma_{0}$.

Note that during the statistical process (i.e., counting $N_{p}$ ), we asign two weighting factors $\left(w_{1}, w_{2}\right)$ to each planetesimal (the tracked test particles), where $w_{1}=a_{p 0}^{-1 / 2}$ leading to an initial surface density profile of $\Sigma_{0} \propto a_{p 0}^{-3 / 2}$ as in MMSN, and $w_{2}=4.2$ if $a_{p 0}>2.7$ AU otherwise $w_{2}=1$, accounting for the solid density enhancement beyond the snow line. The gas disk model is the CGD model as described in $\$ 2.1$.

We conduct a large set of simulations in which we vary the stellar mass ratios $\left(q=M_{B} / M_{A}=\right.$ $0.2,0.5,1.0)$, binary orbital semi-major axes $\left(a_{B}=10,20,50,100,200 \mathrm{AU}\right)$, binary orbital eccentricities $\left(e_{B}=0,0.3,0.6\right)$, binary orbital inclinations $\left(i_{B}=\right.$ $\left.10^{\circ}, 30^{\circ}, 50^{\circ}, 60^{\circ}, 70^{\circ}, 80^{\circ}, 90^{\circ}, 100^{\circ}, 110^{\circ}, 120^{\circ}, 130^{\circ}, 150^{\circ}\right)$, and the planetesimal radial sizes $\left(R_{p}=5,100 \mathrm{~km}\right)$. For the convenience of describing the results, we first define a standard case, then we vary one of parameters of the standard case each time to see the effects of the parameter on the result.

\section{2. the Standard Case}

For the parameters of the standard case, we choose $q=$ $0.5, a_{B}=50 \mathrm{AU}, e_{B}=0.3, i_{B}=60^{\circ}$, and $R_{p}=5 \mathrm{~km}$. The results of the standard case (highlighted with thick blue axes) are shown in the second panel of the righthand column of Fig 9 and in the middle panels of Fig 10 and Fig 11] the results of which can be summarized as follows.

- In the first $2 \times 10^{4} \mathrm{yr}$, the planetesimal disk profile varies a lot. In the outer region the profile becomes very noisy with modest fluctuation around $\Sigma_{0}$. This complicated structure is due to the stepwise jumping as shown in Fig 6 (see the bottomright panel) and the chaotic behavior near the outer boundary (see Fig 7). The modest density surge near $\sim 2.7 \mathrm{AU}$ is due to the initial density jump at the "snowline" $a_{i c e}=2.7 \mathrm{AU}$. In contrast, in the inner region $(\sim 0.3 \mathrm{AU})$ a significant density enhancement (up to $\Sigma / \Sigma_{0} \sim 10$ ) is observed, caused by the planetesimal jumping shown in Fig 6 (see the bottom-middle panel) and Fig 7.

- At times $\sim 10^{5} \mathrm{yr}$, the planetesimal disk appears truncated near $a_{c}$ (Eq[17). Within $\sim a_{c}$, the entire surface density is enhanced by 1 order of magnitude, i.e., $\Sigma / \Sigma_{0} \sim 10$, while beyond $\sim a_{c}$, the surface density is entirely depleted down to $\Sigma / \Sigma_{0}<0.1$. This result is expected from Fig 7 which shows that most, if not all, planetesimals jump into the inner region within $a_{c}$.

- At later times $\left(t \gtrsim 10^{5} \mathrm{yr}\right)$, the planetesimal disk seems to reach a quasi-equilibrium state: the surface density maintains a " 7 " (daleth) shape profile with the sharp outer boundary from $\sim a_{c}$ slowly moving inward. This "orderly" evolution is expected because, as show in Fig 6 ( $\$ 3.3)$, once the planetesimal has completed a jump within $a_{c}$, its $\hat{e}$ and/or $\hat{i}$ would no longer be excited by the binary perturbations and thus it undergoes slow and smooth inward migration. In other words, the planetesimal's inward migration has transited from $\mathrm{B} 1$ regime to $\mathrm{SB}$ and finally to the $\mathrm{S}$ regime as shown in Fig 4 ( $(3.1)$. As the inward migration rate is inverse proportional to its semimajor axis (see $\$ 3.1$ ) in the $\mathrm{S}$ regime, the surface density of planetesimals will then decrease from inside to outside on a long timescale (depending on the gas density and the planetesimal size).

\subsection{Parameter Exploration}

Through comparisons to the standard case, we will discuss the effects of the modeling parameters, such as $i_{B}$, $a_{B}, e_{B}, q_{B}, R_{p}$ and $f_{d}$.

\subsubsection{Effects of $i_{B}$}

We vary the parameter $i_{B}$ of the standard case from $10^{\circ}$ to $150^{\circ}$ but hold all other parameters constant. Our results are plotted in Fig 9 (the standard case is in the second panel of the right-hand column of Fig.9. outlined in bold), and can be summarized as follows.

- Kozai-On case $\left(40^{\circ}<i_{B}<140^{\circ}\right)$. Similar to the standard case, the planetesimal disk reaches a quasi-equilibrium state with a " 7 " (daleth) profile on a timescale of $\sim 10^{5} \mathrm{yr}$ (This process is a little bit quicker for larger $i_{B}$ cases). In all cases, the planetesimals pile up within $a_{c}$, leading to an average surface density enhancement up to one order of magnitude, i.e., $\Sigma / \Sigma_{0} \sim 10$. Note, results of the prograde cases $\left(i_{B}=50^{\circ}, 60^{\circ}, 70^{\circ}, 80^{\circ}\right)$ and their corresponding retrograde cases $\left(i_{B}=\right.$ $130^{\circ}, 120^{\circ}, 110^{\circ}, 120^{\circ}$ ) are very similar (see also in Fig 8). The only slight difference lies in the marginally quicker clearing of the outer disk.

- Kozai-Off case $\left(i_{B}=30^{\circ}, 150^{\circ}\right)$. In contrast to the Kozai-on case, the planetesimal disk seems to evolve to a unimodal profile on a timescale of $\sim$ $10^{5} \mathrm{yr}$. This unimodal profile is maintained with a peak enhancement $\left(\Sigma / \Sigma_{0} \sim 10\right)$ at $\sim a_{c}$, slowly moving inward over time. Another feature which distinguishes this from the Kozai-On case is that the disk will not be significantly truncated with respect to the initial disk. Note again, the prograde case $\left(i_{B}=30^{\circ}\right)$ and its corresponding retrograde case $\left(i_{B}=150^{\circ}\right)$ have very similar results.

- Near-coplanar case $\left(i_{B}=10^{\circ}\right)$. Only modest density enhancement occurs in $\sim 1.5-2.7$ AU because planetesimals pass through the "snowline" $\left(a_{i c e}=2.7 \mathrm{AU}\right)$.

\subsubsection{Effects of $a_{B}$}

We vary the parameter $a_{B}$ of the standard case from 10 to 200 AU but hold all other parameters constant. Results are plotted in Fig [10 (the standard case is in the middle panel highlighted with blue bold axes), which are summarized as the following.

- Similar to the standard case, in all cases (except the $a_{B}=200$ AU case) the planetesimal disks reach a 
quasi-equilibrium, i.e. a "ד" (daleth) shape surface density profile with all planetesimals piling-up in a tight, dense inner region which is truncated near $a_{c}$. In the $a_{B}=200 \mathrm{AU}$ case, the planetesimal disk is not truncated and there are still many planetesimals left beyond $20 \mathrm{AU}$ after $10^{6} \mathrm{yr}$. This is mainly due to the very low gas density there (thus weak gas drag) and due to the long binary separation (thus weaker perturbations). In addition, the surface density enhancements in the $a_{B}=10$ and 20 AU cases are significantly lower $\left(\Sigma / \Sigma_{0} \sim 5\right)$ as compared to the standard case $\left(a_{B}=50 \mathrm{AU}\right.$, $\Sigma / \Sigma_{0} \sim 10$ ) because the initial planetesimal disks are much smaller (thus less total planetesimals) in such close binary systems.

- The time for the planetesimal disk to reach a quasiequilibrium ("T-like shape) surface density profile (we define it as $t_{\text {pile }}$ ) is very sensitive to $a_{B}$. Empirically and to a first order of estimation, $t_{\text {pile }}$ is roughly equal to the secular perturbation timescale at $a_{c}$. Substituting the semimajor axis in Eqn 11] with Eqn 17 pile can be expressed as

$$
\begin{gathered}
t_{\text {pile }} \sim 28.7 \times\left(\frac{a_{B}}{\mathrm{AU}}\right)^{2}\left(1-e_{B}^{2}\right) \\
\left(\frac{M_{A}}{M_{\odot}}\right)^{1 / 6}\left(\frac{M_{B}}{M_{\odot}}\right)^{-2 / 3} \mathrm{yr},
\end{gathered}
$$

which depends mainly on $a_{B}$ and weakly on $e_{B}$, $M_{A}$ and $M_{B}$. For $a_{B}>200 \mathrm{AU}$, the pile timescale $t_{\text {pile }}>1$ Myr, which is comparable or even longer than the lifetime of the gas disk. Therefore, the jump-piling effects would be very limited for binary systems with $a_{B}>200 \mathrm{AU}$.

\subsubsection{Effects of $e_{B}, q_{B}, R_{p}$ and $f_{d}$}

The results are shown in Fig.11 with the standard case located in the middle panel with blue bold axes. We can obtain the effects of $e_{B}$ and $q_{B}$ through the comparison of the three middle vertical panels and the comparison of the three horizontal panels, respectively. In addition, we can compare the cases of $R_{p}=100 \mathrm{~km}$ and $R_{p}=1$ $\mathrm{km}$ (the two bottom-corner panels) to the standard case (the middle panel with blue bold axes) for the effects of $R_{p}$. Since increasing the planetesimal radial size $\left(R_{p}\right)$ is equivalent to decreasing the gas density $\left(f_{d}\right)$ from a dynamical view, thus we obtain the effect of $f_{d}$ at the same time.

- Effects of $e_{B}\left(q_{B}\right)$. Increasing $e_{B}\left(q_{B}\right)$ leads to quicker evolution of the disk density profile (shorter $t_{\text {pile }}$ as also seen the dependence in Eqn[19] and slightly lower surface density enhancement $\left(\Sigma / \Sigma_{0}\right)$ due to the stronger binary perturbation and smaller initial planetesimal disk size.

- Effects of $R_{p}\left(f_{d}\right)$. Increasing $R_{p}$ (decreasing $f_{d}$ ) is a double-edged sword when it comes to the planetesimal pile-up. On the one hand, it directly reduces the gas drag acceleration on the planetesimals and thus slows their "jumping processes" and inhibits the planetesimal piling (see the leftover material in the outer disk of the $R_{p}=100 \mathrm{~km}$ case in Fig 11). On the other hand, once planetesimals have finished their jump into the inner region, they will experience much stronger braking of their further inward migration because of the larger $R_{p}$ (smaller $f_{d}$ ), which favors the planetesimal pile-up (see the nearly fixed boundary around $a_{c}$, and the progressive increase of the surface density within $a_{c}$ in the $R_{p}=100 \mathrm{~km}$ case). The opposite trend can be found by decreasing $R_{p}$ or increasing $f_{d}$ (see the case of $R_{p}=1 \mathrm{~km}$ ).

\subsection{Analytic Estimate of $\Sigma / \Sigma_{0}$}

As we have shown above, the inward migration or jumping process always lead to the formation of a compact, dense disk with an outer boundary around $a_{c}$. Therefore, the surface density enhancement in the inner region can be estimated by assuming that all of the material initially having $a>a_{c}$ is transported to $a<a_{c}$, leading to an enhancement factor

$$
\frac{\Sigma}{\Sigma_{0}} \sim \frac{\int_{a_{i n}}^{a_{d}} 2 \pi r \Sigma_{0} d r}{\int_{a_{i n}}^{a_{c}} 2 \pi r \Sigma_{0} d r}
$$

where $a_{i n}$ (here set as $0.1 \mathrm{AU}$ ) is the inner boundary of the planetesimal disk and $a_{d}$ is the initial outer boundary following Holman \& Wiegert (1999). Considering a power law disk profile as $\Sigma_{0} \propto r^{\alpha}(\alpha \neq 2)$, then Eq20 can be rewritten as

$$
\frac{\Sigma}{\Sigma_{0}} \sim \begin{cases}\frac{a_{d}^{2+\alpha}-a_{i n}^{2+\alpha}}{a_{c}^{2+\alpha}-a_{i n}^{2+\alpha}} & \text { if } a_{c}<a_{d}<a_{i c e}, \\ \frac{a_{i c e}^{2+\alpha}-a_{i n}^{2+\alpha}+f_{i c e}\left(a_{d}^{2+\alpha}-a_{i c e}^{2+\alpha}\right)}{a_{c}^{2+\alpha}-a_{i n}^{2+\alpha}} & \text { if } a_{c}<a_{i c e}<a_{d}, \\ \frac{a_{i c e}^{2+\alpha}-a_{i n}^{2+\alpha}+f_{i c e}\left(a_{d}^{2+\alpha}-a_{i c e}^{2+\alpha}\right)}{a_{i c e}^{2+\alpha}-a_{i n}^{2+\alpha}+f_{i c e}\left(a_{c}^{2+\alpha}-a_{i c e}^{2+\alpha}\right)} \text { if } a_{i c e}<a_{c}<a_{d},\end{cases}
$$

where $a_{i c e}$ is the location of snow line and $f_{i c e}$ is the solid density enhancement beyond the snow line. Following Ida \& Lin (2004), we take $a_{i c e}=2.7$ AU and $f_{i c e}=4.2$.

Fig 12 shows the results of Eq21 We see that the analytical estimates are consistent with the N-body simulation results by comparing the red solid curve of Fig 12 to Fig[10. Piling effects are most significant for binary systems with $a_{B} \sim 100 \mathrm{AU}$ and for planetesimal disks with flatter initial disk profiles. While the profile we consider in detail $(\alpha=3 / 2)$ have surface density enhancements $\sim 10$, for disk profiles flatter than $\Sigma_{0} \propto r^{-3 / 4}$, the surface density enhancement can be around 2 orders of magnitude.

\section{DISCUSSIONS}

\subsection{Implications for Planet Formation in Highly Inclined Systems}

In this paper, we have shown, for the highly inclined $\left(i_{B} \gtrsim 30^{\circ}\right)$ binary systems, that planetesimals from the outer disk can "jump" inwards and pile up in the inner disk. The typical result of this jumping-piling process is the formation of a smaller and denser planetesimal disk around the primary, with the truncation boundary near $a_{c}$ (Eqn 17), within which the surface density enhancement is up to $\Sigma / \Sigma_{0} \sim 10$. This significant change in the planetesimal disk will definitely affect the subsequent planet-formation processes-planetesimal growth, 
and the final composition and configuration of the planetary system.

The two most important parameters which govern planetesimal growth are, first, the relative velocities $\left(V_{\text {rel }}\right)$ among planetesimals, and second, their collision rate. The former determines the outcome of planetesimal-planetesimal collisions, i.e., accretion or erosion; while the latter controls the speed of planetesimal accretion and/or erosion. We investigate in Fig 13 the evolution of these two parameters during the jumping-piling process for the standard binary configuration $\left(M_{A}=M_{\odot}, q_{B}=M_{B} / M_{A}=0.5, a_{B}=50\right.$ $\left.\mathrm{AU}, e_{B}=0.3, i_{B}=60^{\circ}\right)$. In Fig[13, 10,000 planetesimals with a centered Gaussian size distribution between $R_{p}=1 \mathrm{~km}$ and $R_{p}=10 \mathrm{~km}$, are initially randomly distributed between 0.3 AU and $10 \mathrm{AU}$ (flat distribution) with near circular and coplanar orbits. As can be seen, in the first $\sim 10^{4} \mathrm{yr}$, the average $V_{\text {rel }}$ has a steep increase up to $10^{3} \mathrm{~ms}^{-1}$ because of the strong binary perturbations, and the collision rate is relatively low because of the large spread of planetesimals at the beginning. After this point $\left(t \sim 10^{4} \mathrm{yr}\right)$, planetesimals begin to jump inwards and pile up in the inner region (within $\sim 1.5 \mathrm{AU}$ ), leading to a surface density enhancement of up to one order of magnitude, a collision rate enhancement of up to three orders of magnitude, and a reduction of average $V_{\text {rel }}$ down to $40-50 \mathrm{~ms}^{-1}$. If we adopt a velocity of $50-100 \mathrm{~ms}^{-1}$ as the threshold for planetesimal erosion as in (Marzari et al. 2009), then we see that the jumping-piling process moves most planetesimals from an erosional regime to an accretional regime. For this reason, we expect fast planetesimal growth to occur in the inner region.

In fact, everything (such as $V_{\text {rel }}, \hat{e}$, and $\hat{i}$ of planetesimals) within $a_{c}$, is getting close to that in single star cases. For example, after planetesimals have completed the jumping process as shown in Fig 6, their eccentricities $(\hat{e})$ are all below $10^{-3}$, which are comparable to that in single star cases (see Eqn.(10) of Kokubo \& Ida $(2000)$ ) and their inward drifts transition to the SB or S regime. Therefore, the process of planetesimal growth within $a_{c}$ would probably follow a similar way as in single star systems. Such a process leads eventually to the formation of planetary embryos (via the runaway and oligarchic growth processes), resulting in a number of embryos which have isolated themselves from one another due to the accretion of all the planetesimals in an annular feeding zone around themselves. This embryo isolation mass is given by (Ida \& Lin 2004)

$$
M_{\text {iso }} \sim 0.16 f_{d}^{3 / 2} f_{\text {ice }}^{3 / 2}\left(\frac{\Sigma}{\Sigma_{0}}\right)^{3 / 2}\left(\frac{a}{\mathrm{AU}}\right)^{3 / 4} \quad \mathrm{M}_{\oplus}
$$

where $f_{\text {ice }} \sim 4.2$ denotes the solid density enhancement beyond the ice line $a_{i c e}=2.7 \mathrm{AU}$, and $\Sigma / \Sigma_{0}$ denotes the solid surface density enhancement relative to MMSN.

Clearly, in a MMSN, at 1 AU the isolation mass is $0.16 M_{\oplus}$, leading to the idea that in the Solar System there may have been a chain of such $\sim$ Mars-mass embryos in the terrestrial planet region, which subsequently went on to collisionally evolve in a chaotic growth phase.

However, in the binary scenario we are considering, we have seen typical surface density enhancements of $\Sigma / \Sigma_{0} \sim 10$ (can be larger for flatter disk profiles as shown in Fig 12), leading to an isolation mass at $1 \mathrm{AU}$ $\sim 5 \times f_{d}{ }^{3 / 2} \mathrm{M}_{\oplus}$. Moreover, the initial surface density of the disk could be above that of the MMSN, so it is entirely plausible to have $f_{d}=1-3$, giving isolation masses at $1 \mathrm{AU}$ in the range $5-26 \mathrm{M}_{\oplus}$ and typical separations $10 r_{H} \sim 0.25-0.43 \mathrm{AU}$.

From the first order estimation above, one could anticipate that in the highly dense inner-disk regions of these inclined binary systems, several giant embryos could form. If these cores grew before the depletion of the gas disk, then several gas-giants could start to form, otherwise, a series of stalled super earths/neptunes may form. In addition, as the planetesimal disk is severely truncated after the jumping-piling process, it is highly probable that such planets would be born in a dynamically compact region with a short stability timescale (Chatteriee et al. 2008). After the depletion of the gas disk, the final configuration of the planetary system would then be further shaped by dynamical evolution mechanisms such as planetplanet scattering (Rasio \& Ford 1996; Ford et al. 2001; Malmberg et al. 2007), Kozai migration (Wu \& Murrav 2003; Fabrycky \& Tremaine 2007; Takeda et al. 2009) and secular chaos (Michtchenko et al. 2006; Lithwick \& Wu 2010; Wu \& Lithwick 2010).

\subsection{Limitations}

First, as presented in $\S 2$, we adopt a very simplified gas disk model (CGD) where the gas is in a circular orbit in the circumprimary mid-plane without feeling the companion star as it is in a single star system. In reality, the gas disk structure should be significantly modified by the binary perturbations. Nevertheless, we found that the planetesimal dynamical behaviors are very similar under different models of disk structure for highly inclined $\left(30^{\circ} \lesssim i_{B} \lesssim 150^{\circ}\right)$ binary systems .

Second, the growth and/or erosion of planetesimals due to mutual collisions are not considered in the present paper. Beyond $a_{c}$, planetesimals are excited onto orbit with large inclinations, leading to their collisional timescale becoming longer than $10^{6} \mathrm{yr}$ (according to Eqn. 12 of Xie et al. 2010), which is larger than the piling timescale $t_{\text {pile }}$ (Eqn[19). Therefore, outer planetesimals can jump and pile up in the inner region before they collide with one another. On the other hand, in the piling region $\left(<a_{c}\right)$, planetesimals may grow up quickly if collisions are considered because of the very high collision rate and low relative velocities as shown in Fig.13. As they grow up, their inward migrations slow down, which can make stronger piling effect in the inner region.

Third, in order to estimate the degree of planetesimal piling effects, we have assumed that all planetesimals have already been born before the jumping-piling effect takes place. This assumption is reasonable only if the planetesimals birth rate is very high over the whole disk. However, some theoretical and observational works (Cuzzi \& Weidenschilling 2006; Scott 2007; Natta et al. 2007; Chauvin et al. 2010) suggest that such a transition process, from dust to planetesimal, may take as long as a few $10^{6}$ years, which is comparable to or even larger than the piling timescale $t_{\text {pile }}$ (Eqn[19). In such a case, much smaller, maybe mm-sized, particles pilingup through other mechanisms (Youdin \& Chiang 2004) 
should be considered.

Finally, perhaps the main uncertainty in the planetesimal "jumping-piling" effect comes from not including the effect of gas gravity on the planetesimals, i.e. we have neglected disk self-gravity. One possible effect of gas gravity might be that it can pull highly inclined planetesimals back towards the mid-plane of the gas disk, so that the Kozai effect (and hence the "jumping-piling" effect) might be inhibited (Fragner et al. 2011). However this effect usually happens for massive gas disk. For the standard case we are considering in this paper $\left(a_{B}=50\right.$ $\mathrm{AU})$, the disk mass should be larger than $\sim 6 \mathrm{MMSN}$ to switch off the Kozai effect as shown in the Fig.16 of Fragner et al. (2011). For a nominal disk with 1 MMSN, we thus expect that the "jumping-piling" process will still be efficient even if the disk gravity is included. In fact, a jumping trend of planetesimal semimajor axis can be discerned in Fig.11 of Fragner et al. (2011). In addition, as is effectively shown in the bottom-right panel of Fig 11 in our paper, the "jumping-piling" process is still efficient even for a very tenuous gas disk of less than 0.05 MMSN. Therefore, it is plausible that the planetesimal "jumping-piling" process described in this paper would be most important during the gas dissipation phase.

\section{SUMMARY}

In this paper, we have investigated the intermediate stage of planet formation - from planetesimals to planetary embryos - in highly inclined binary systems with a focus on the effects of gas drag on planetesimal orbits, especially the evolution in the semimajor axis distribution.

First, we justify our choice of a simplified circular gas disk (CGD) for modeling gas drag force in highly inclined cases. Through numerical tests and comparisons (see Fig 2 and Fig, we have shown that eccentricity and pericenter precession of the gas disk are not important to the effects of gas drag on a planetesimal for binary systems with inclination $30^{\circ} \lesssim i_{B} \lesssim 150^{\circ}$. The major factor in determining the magnitude of the gas drag forces is the mutual inclination $\hat{i}$ between the planetesimal and the gas disk, which is mainly controlled by $i_{B}$ and is independent of the specific disk model.

Next, we analyze the dynamics of individual planetesimals under the influence of gas drag which leads to the inward migration of plantesimals. Depending on the dominant mechanism which drives the planetesimal eccentricities and inclinations, very different migration behaviors can take place. There are three main regimes to consider (see Fig 4): Regime S, where the situation is close to that in single star systems; Regime B where inward migration is dominated by the binary's perturbations, and an intermediate transition regime $\mathrm{SB}$. In addition, regime $\mathrm{B}$ can be divided into three sub-regimes, two of which, the Kozai-off regime and the Kozai-on regime, are studied in detail in the present paper.

For the Kozai-off regime $\left(i_{B}<39.2^{\circ}\right.$ or $\left.i_{B}>140.8^{\circ}\right)$, planetesimal inward migration is determined only by the excitation and evolution of its orbital inclination $(\hat{i})$.
There is a turnover semimajor axis $\left(a_{c}\right.$, see Eqn 17 and Fig (5), where planetesimals transit from a fast inward migration (regime B) to a slow one (regime $\mathrm{SB}$ or $\mathrm{S}$ ). The value of $a_{c}$ is independent of planetesimal initial semimajor axis $\left(a_{p 0}\right)$, and it can be estimated by equating the timescale of secular perturbation $\left(t_{s p}\right.$, see Eqn 11) and the timescale of gas drag damping $\left(t_{d p}\right.$, see Eqn 15$)$.

For the Kozai-on regime $\left(39.2^{\circ}<i_{B}<140.8^{\circ}\right)$, planetesimals can be excited to orbits with high eccentricities and high inclinations (or even become retrograde with respect to the gas disk). In such a case, planetesimal inward migration can be very sudden. Planetesimals in the outer disk can directly jump into the inner disk where they then slow their inward migration as their orbits circularize and become coplanar due to strong gas drag damping (see Fig 6). Unlike the Kozai-off case, the turnover semimajor axis for this migration transition is no longer independent of $a_{p 0}$, but it is always located in a range between $a_{c(l o w)}$ (see, Fig[7 and Fig 8) and $a_{c}$.

We then studied the behavior of a swarm of planetesimals for both Kozai-on and Kozai-off cases with an exploration of many parameters (see Fig 9, Fig.10, and Fig 11), including the binary mass ratio $\left(q_{B}\right)$, semimajor axis $\left(a_{B}\right)$, eccentricity $\left(e_{B}\right)$, inclination $\left(i_{B}\right)$, gas density scale $\left(f_{d}\right)$ and planetesimal radial size $\left(R_{p}\right)$. A robust result is that planetesimals migrate/jump inwards and pile up in the inner region, leading to a smaller and denser planetesimal disk with a truncatation boundary near $a_{c}$ and a surface density enhancement or order $\Sigma / \Sigma_{0} \sim 10$. The timescale for such a "jumping-piling" process to operate, $t_{\text {pile }}$ (see Eqn[19), is mainly dependent on $a_{B}$.

Applying such "jumping-piling" effects to planet formation, we expect a growth-favored region for planetesimals in the inner disk (within $a_{c}$ ), where collision rates are high and relative velocities are low (see Fig 13). Such conditions may lead to the formation of embryos sufficiently massive to undergo runaway gas accretion (provided they form prior to the dissipation of the gas disk).

This work, focusing only on the effect of gas drag on a planetesimal, is our first step towards understanding planet formation in highly inclined binary systems. Future work needs to account for important physical factors such as the effects of gas disk gravity, planetesimal accretion/fragmentation, and the time at which planetesimals emerge and the jump-piling process begins.

\section{ACKNOWLEDGMENTS}

This work was supported by the National Natural Science Foundation of China (Nos.10833001, 10778603 and 10925313), the National Basic Research Program of China(No.2007CB814800), NSF with grant AST0705139 and 0707203 , NASA with grant NNX07AP14G and NNX08AR04G, W.M. Keck Foundation, Nanjing University and also University of Florida. J.-W. Xie was also supported by the China Scholarship Council and China Ministry of Education. M. J. Payne was also supported by the NASA Origins of Solar Systems grant NNX09AB35G and the University of Florida's College of Liberal Arts and Sciences.

\section{REFERENCES}

Adachi, I., Hayashi, C., \& Nakazawa, K. 1976, Progress of Theoretical Physics, 56, 1756
Armitage, P. J. 2010, Astrophysics of Planet Formation, by Philip J. Armitage, pp. 294. ISBN 978-0-521-88745-8

(hardback). Cambridge, UK: Cambridge University Press, 2010. 
Artymowicz, P., \& Lubow, S. H. 1994, ApJ, 421, 651

Barbieri, M., Marzari, F., \& Scholl, H. 2002, A\&A, 396, 219

Beaugé, C., Leiva, A. M., Haghighipour, N., \& Otto, J. C. 2010, MNRAS, 408, 503

Benz, W., \& Asphaug, E. 1999, Icarus, 142, 5

Blum, J., \& Wurm, G. 2008, ARA\&A, 46, 21

Chiang, E., \& Youdin, A. N. 2010, Annual Review of Earth and Planetary Sciences, 38, 493

Chambers, J. E., \& Wetherill, G. W. 1998, Icarus, 136, 304

Chambers, J. E. 1999, MNRAS, 304, 793

Chambers, J. E. 2010, Icarus, 208, 505

Chatterjee, S., Ford, E. B., Matsumura, S., \& Rasio, F. A. 2008, ApJ, 686, 580

Chauvin, G., Beust, H., Lagrange, A. -., \& Eggenberger, A. 2010, arXiv:1009.5851

Cieza, L. A., et al. 2009, ApJ, 696, L84

Correia, A. C. M., et al. 2008, A\&A, 479, 271

Cuzzi, J. N., \& Weidenschilling, S. J. 2006, Meteorites and the Early Solar System II, 353

Cuzzi, J. N., Hogan, R. C., \& Shariff, K. 2008, ApJ, 687, 1432

Desidera, S., \& Barbieri, M. 2007, A\&A, 462, 345

Duchêne, G., Delgado-Donate, E., Haisch, K. E., Jr., Loinard, L., \& Rodríguez, L. F. 2007, Protostars and Planets V, 379

Duquennoy, A., \& Mayor, M. 1991, A\&A, 248, 485

Eggenberger, A., Udry, S., \& Mayor, M. 2004, A\&A, 417, 353

Eggenberger, A. 2010, EAS Publications Series, 42, 19

Fabrycky, D., \& Tremaine, S. 2007, ApJ, 669, 1298

Ford, E. B., Havlickova, M., \& Rasio, F. A. 2001, Icarus, 150, 303

Fragner, M.M., Nelson, R.P., \& Kley, W. 2011, A\&A, inpress

Goldreich, P., \& Ward, W. R. 1973, ApJ, 183, 1051

Guedes, J. M., Rivera, E. J., Davis, E., Laughlin, G., Quintana, E. V., \& Fischer, D. A. 2008, ApJ, 679, 1582

Haghighipour, N. 2010, Astrophysics and Space Science Library, 366 ,

Hale, A. 1994, AJ, 107, 306

Hatzes, A. P., Cochran, W. D., Endl, M., McArthur, B., Paulson, D. B., Walker, G. A. H., Campbell, B., \& Yang, S. 2003, ApJ, 599, 1383

Hayashi, C. 1981, Progress of Theoretical Physics Supplement, 70,35

Heppenheimer, T. A. 1978, A\&A, 65, 421

Holman, M. J., \& Wiegert, P. A. 1999, A.J, 117, 621

Ida, S., \& Lin, D. N. C. 1996, AJ, 112, 1239

Ida, S., \& Lin, D. N. C. 2004, ApJ, 604, 388

Jensen, E. L. N., Mathieu, R. D., Donar, A. X., \& Dullighan, A. 2004, ApJ, 600, 789

Johansen, A., Brauer, F., Dullemond, C., Klahr, H., \& Henning, T. 2008, A\&A, 486, 597

Kokubo, E., \& Ida, S. 1996, Icarus, 123, 180

Kokubo, E., \& Ida, S. 1998, Icarus, 131, 171

Kokubo, E., \& Ida, S. 2000, Icarus, 143, 15

Kokubo, E., Kominami, J., \& Ida, S. 2006, ApJ, 642, 1131

Kley, W. 2000, IAU Symposium, 200, 211P

Kley, W., \& Nelson, R. P. 2008, A\&A, 486, 617

Kortenkamp, S. J., Wetherill, G. W., \& Inaba, S. 2001, Science, 293, 1127

Kozai, Y. 1962, AJ, 67, 591

Larwood, J. D., Nelson, R. P., Papaloizou, J. C. B., \& Terquem, C. 1996, MNRAS, 282, 597

Lee, J. W., Kim, S.-L., Kim, C.-H., Koch, R. H., Lee, C.-U., Kim, H.-I., \& Park, J.-H. 2009, AJ, 137, 3181

Levison, H. F., \& Agnor, C. 2003, AJ, 125, 2692

Lissauer, J. J. 1993, ARA\&A, 31, 129

Lithwick, Y., \& Wu, Y. 2010, arXiv:1012.3706

Malmberg, D., Davies, M. B., \& Chambers, J. E. 2007, MNRAS, 377, L1

Marzari, F., \& Scholl, H. 2000, ApJ, 543, 328

Marzari, F., Thébault, P., \& Scholl, H. 2009, A\&A, 507, 505

Mathieu, R. D., Ghez, A. M., Jensen, E. L. N., \& Simon, M. 2000, Protostars and Planets IV, 703
Michtchenko, T. A., Ferraz-Mello, S., \& Beaugé, C. 2006, Icarus, 181,555

Miguel, Y., Guilera, O. M., \& Brunini, A. 2010, arXiv:1010.5061

Monin, J.-L., Ménard, F., \& Peretto, N. 2006, A\&A, 446, 201

Monin, J.-L., Clarke, C. J., Prato, L., \& McCabe, C. 2007,

Protostars and Planets V, 395

Mugrauer, M., \& Neuhäuser, R. 2009, A\&A, 494, 373

Natta, A., Testi, L., Calvet, N., Henning, T., Waters, R., \& Wilner, D. 2007, Protostars and Planets V, 767

Paardekooper, S.-J., Thébault, P., \& Mellema, G. 2008, MNRAS, 386, 973

Paardekooper, S.-J., \& Leinhardt, Z. M. 2010, MNRAS, 403, L64

Pascucci, I., Apai, D., Hardegree-Ullman, E. E., Kim, J. S.,

Meyer, M. R., \& Bouwman, J. 2008, ApJ, 673, 477

Pichardo, B., Sparke, L. S., \& Aguilar, L. A. 2005, MNRAS, 359, 521

Pringle, J. E. 1981, ARA\&A, 19, 137

Prša, A., et al. 2011, AJ, 141, 83

Quintana, E. V., Lissauer, J. J., Chambers, J. E., \& Duncan,

M. J. 2002, ApJ, 576, 982

Quintana, E. V. 2004, Ph.D. Thesis,

Quintana, E. V., \& Lissauer, J. J. 2006, Icarus, 185, 1

Quintana, E. V., Adams, F. C., Lissauer, J. J., \& Chambers, J. E. 2007, ApJ, 660, 807

Queloz, D., et al. 2000, A\&A, 354, 99

Rafikov, R. R. 2003, AJ, 125, 942

Rafikov, R. R. 2004, AJ, 128, 1348

Rasio, F. A., \& Ford, E. B. 1996, Science, 274, 954

Safronov, V. S. 1969. Evolution of the Protoplanetary Cloud and Formation of the Earth and Planets. Moscow: Nauka. Eng. trans. NASA TTF-677, 1972

Scholl, H., Marzari, F., \& Thébault, P. 2007, MNRAS, 380, 1119

Scott, E. R. D. 2007, Annual Review of Earth and Planetary Sciences, 35, 577

Sigurdsson, S., Richer, H. B., Hansen, B. M., Stairs, I. H., \&

Thorsett, S. E. 2003, Science, 301, 193

Slawson, R. W., et al. 2011, arXiv:1103.1659

Stewart, S. T., \& Leinhardt, Z. M. 2009, ApJ, 691, L133

Takeda, G., Kita, R., \& Rasio, F. A. 2009, IAU Symposium, 253, 181

Takeuchi, T., \& Lin, D. N. C. 2002, ApJ, 581, 1344

Teiser, J., \& Wurm, G. 2009, MNRAS, 393, 1584

Thébault, P., Marzari, F., \& Scholl, H. 2006, Icarus, 183, 193

Thébault, P., Marzari, F., \& Scholl, H. 2008, MNRAS, 388, 1528

Thébault, P., Marzari, F., \& Scholl, H. 2009, MNRAS, 393, L21

Weidenschilling, S. J. 1977, MNRAS, 180, 57

Weidenschilling, S. J., \& Cuzzi, J. N. 1993, Protostars and

Planets III, 1031

Weidenschilling, S. J. 1997, From Stardust to Planetesimals, 122, 281

Wetherill, G. W., \& Stewart, G. R. 1989, Icarus, 77, 330

Whitmire, D. P., Matese, J. J., Criswell, L., \& Mikkola, S. 1998, Icarus, 132, 196

Wu, Y., \& Murray, N. 2003, ApJ, 589, 605

Wu, Y., \& Lithwick, Y. 2010, arXiv:1012.3475

Xie, J.-W., \& Zhou, J.-L. 2008, ApJ, 686, 570

Xie, J.-W., \& Zhou, J.-L. 2009, ApJ, 698, 2066

Xie, J.-W., Zhou, J.-L., \& Ge, J. 2010a, ApJ, 708, 1566

Xie, J.-W., Payne, M. J., Thébault, P., Zhou, J.-L., \& Ge, J. 2010b, ApJ, 724, 1153

Youdin, A. N., \& Chiang, E. I. 2004, ApJ, 601, 1109

Youdin, A. N., \& Goodman, J. 2005, ApJ, 620, 459

Youdin, A., \& Johansen, A. 2007, ApJ, 662, 613

Youdin, A. N., \& Shu, F. H. 2002, ApJ, 580, 494

Zsom, A., Sandor, Z., \& Dullemond, C. 2010, arXiv:1010.4210

Zucker, S., Mazeh, T., Santos, N. C., Udry, S., \& Mayor, M. 2004, A\&A, 426, 695 


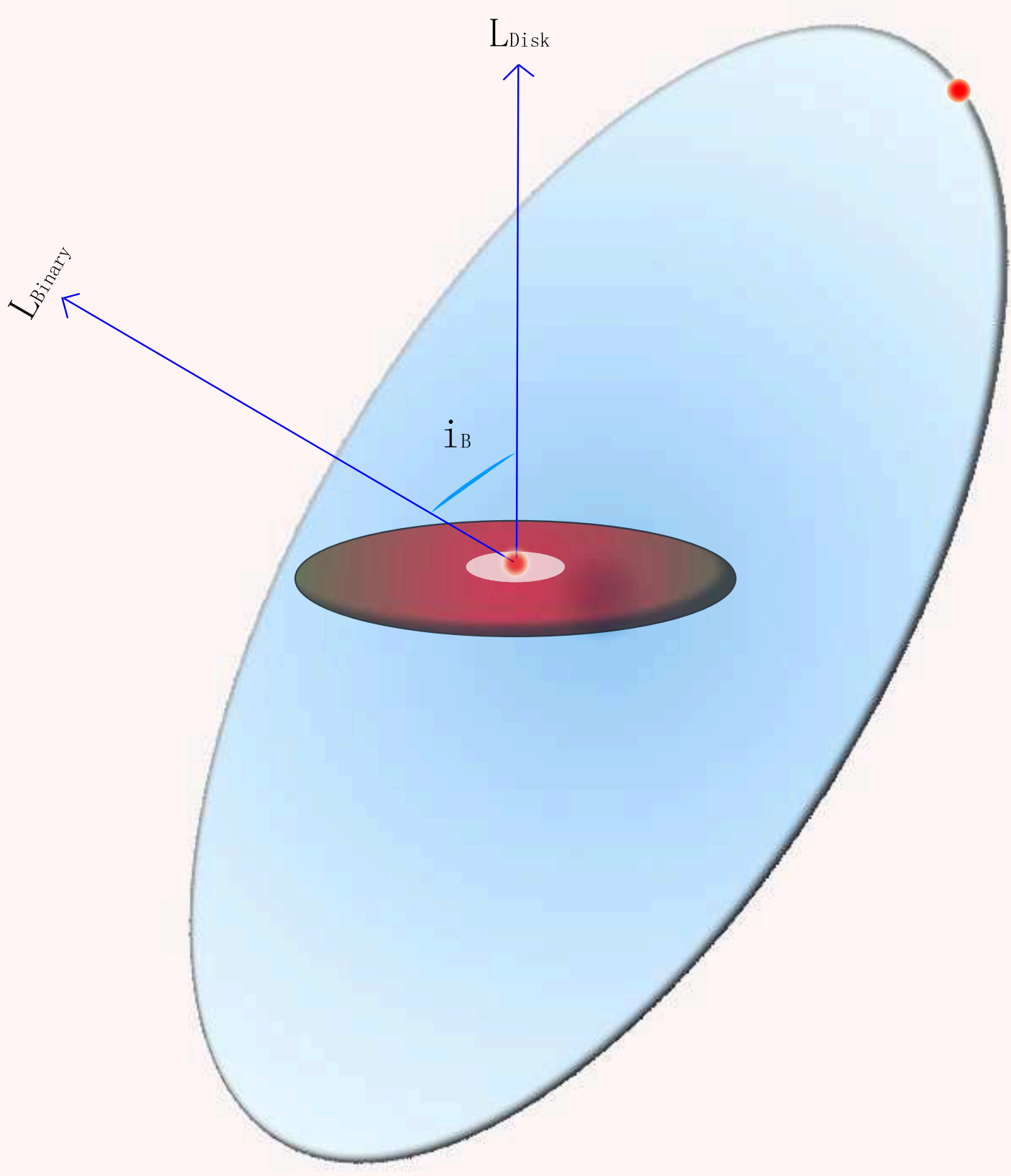

FIG. 1. - Sketch of the orbital configuration of the highly inclined binary system studied in this paper. $L_{D i s k}$ and $L_{B i n a r y}$ are the normals of the gas disk plane and the binary orbital plane, respectively. The angle $i_{B}$ between $L_{\text {Disk }}$ and $L_{B i n a r y}$ corresponds to the mutual inclination of the two planes. 

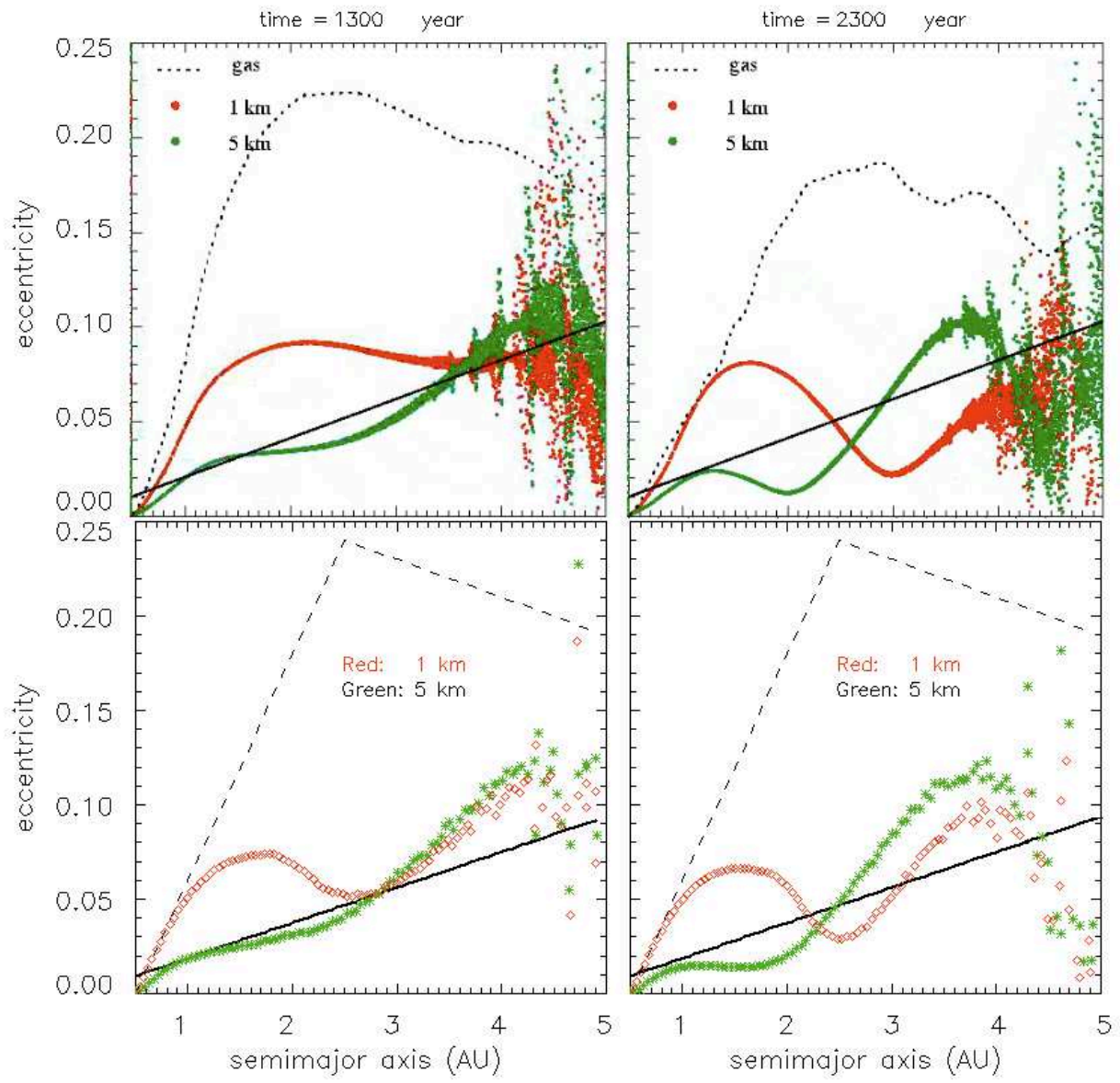

FIG. 2.- Comparison between the results given by the hydrodynamical model (top: Paardekooper et al. (2008)) and the approximate EGD model (bottom). We display snapshots of planetesimal eccentricity vs. semimajor at $\mathrm{t}=1300$ and 2300 yr. The black solid lines indicate the forced eccentricity $e_{f}=\left(5 a e_{B}\right) /\left(4 a_{B}\right)$. The black dashed lines show the eccentricity distribution of the gas. We see that the results from the EGD model can recover the basic features of the results by (Paardekooper et al. (2008), thus providing us with a means to simply and quickly add the main features of the time-consuming hydrodynamical results into a fast N-body code. 

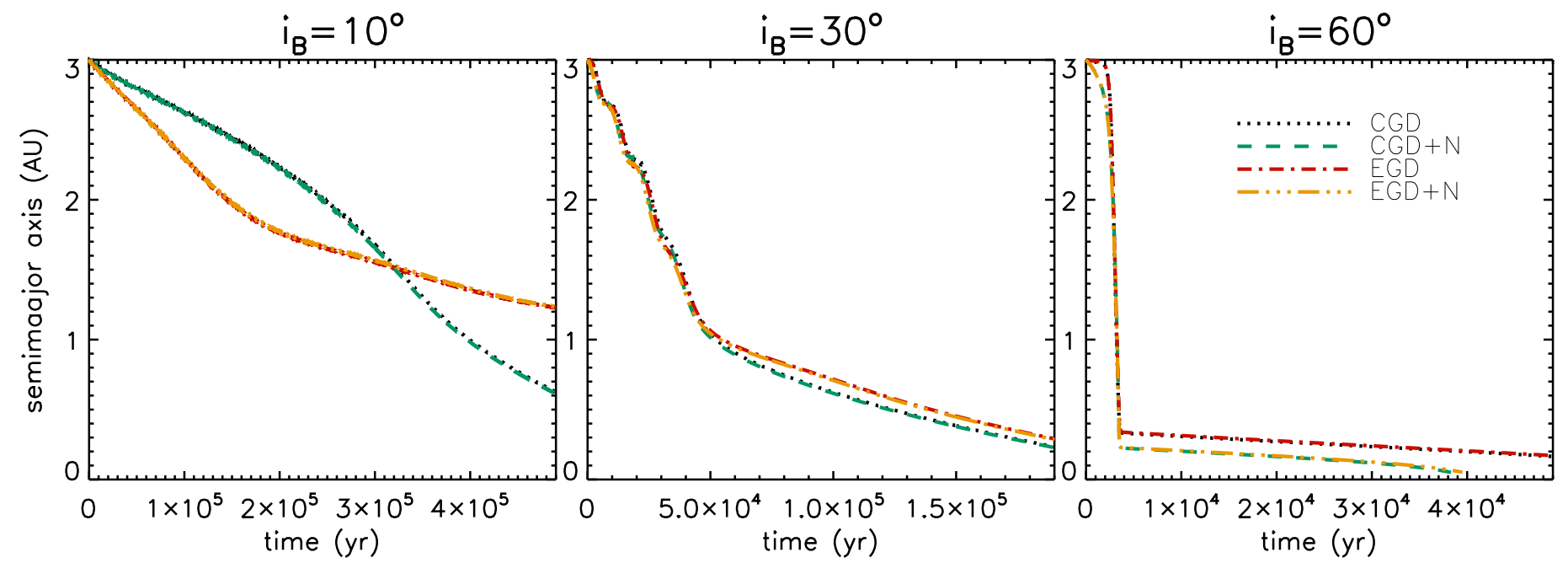

FIG. 3.- Comparison between the results obtained from 4 different disk models (CGD, CGD+N, EGD, EGD+N) for three cases with $i_{B}=10^{\circ}, 30^{\circ}$ and $60^{\circ}$. We plot the evolution of a planetesimal's semi-major axis. We see, for highly inclined cases $\left(i_{B}>30^{\circ}\right)$, the evolution of planetesimal semi-major axis is nearly independent of the eccentricity and nodal precession of the gas. We thus feel justified in using the simple CGD model for the remainder of our investigation due to the fact that we are concentrating on the high inclination cases. A detailed investigation of the planetesimal dynamics, including the reason for large inward jumps is provided in $\S 3$ 


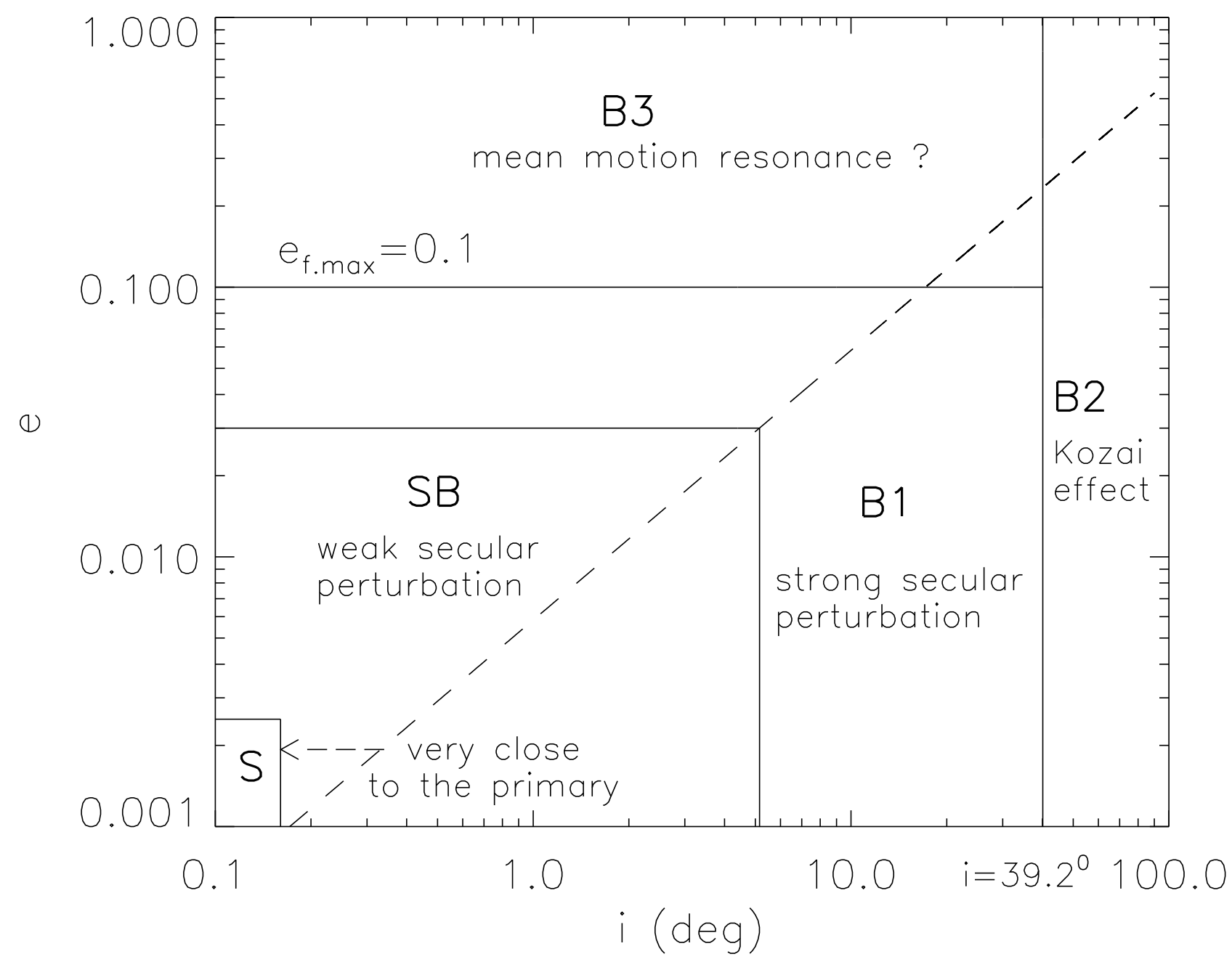

FIG. 4.- Schematic diagram of different regimes for the damping of planetesimal semimajor axis. The damping is dominated by $\eta$ (the sub-Keplerian gas velocity) in region $\mathrm{S}$, the evolution of $i$ and $e$ dominates the gas damping in region $\mathrm{B}$. Region $\mathrm{B}$ is divided into 3 sub-regions depending on the particular mechanism by which $e$ and $i$ are excited; secular perturbations (B1), Kozai effect (B2), and other effects such as mean motion resonance by the companion star (B3). SB is a transition region between S and B, in which contributions from $e, i$ and $\eta$ can all be significant. The dashed line divides the plot into two parts: $e$ has more contribution to the damping of semi-major axis than than $i$ does in the top-left part, while in the bottom-right part the opposite is true. For full details, see the text in $\S 3.1$ 

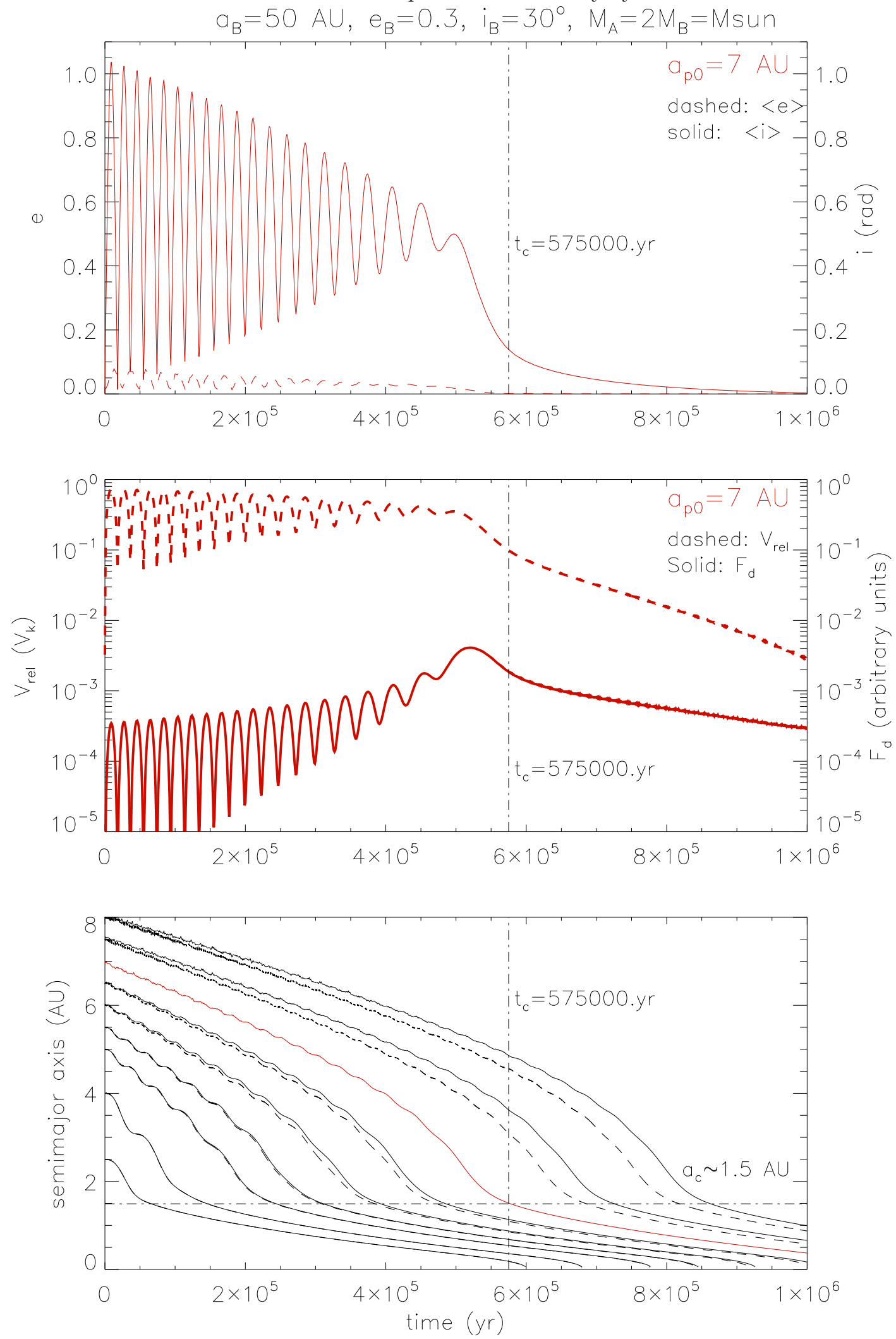

FIG. 5.- The evolution of orbital eccentricity ( $\hat{e}$, dashed, top), inclination ( $\hat{i}$, solid, top), velocity relative to the gas ( $V_{\text {rel }}$ in units of local Keplerian velocity $V_{k}$, dashed, middle), gas drag force $\left(F_{d}\right.$, solid middle), semimajor axis (red solid, bottom) of a planetesimal with $a_{p 0}=7$ $\mathrm{AU}$, and $R_{p}=5 \mathrm{~km}$ under the coupled effects of perturbations from a binary $\left(a_{B}=50 \mathrm{AU}, e_{B}=0.3, i_{B}=30^{\circ}, M_{A}=2 M_{B}=M_{\odot}\right)$ and gas drag from a gas disk $(1 \mathrm{MMSN})$. Planetesimal orbital eccentricities and inclinations are initially set as very small values $\left(<10^{-4}\right)$, and their other initial angular orbital elements are set as random values from 0 to $360^{\circ}$. In the bottom panel, we also plot the results for planetesimals with different initial semi-major axis $a_{p 0}$. The dashed curves are the results of retrograde case $\left(i_{B}=150^{\circ}\right)$ for comparison. We see that all planetesimals' inward migrations transit from a fast regime to a slow one at a similar turn-over semi-major axis, which is independent of $a_{p 0}$. This turn-over semi-major axis can be well described with $a_{c}$ (Eq 17 and see also the horizonal dot-dash line in the bottom panel). Note that $\hat{i}$ is measured relative to the plane of the gas disk rather than to the binary orbital plane. The vertical dot-dash line denotes the time at the turnover point for the case of $a_{p 0}=7 \mathrm{AU}$. 

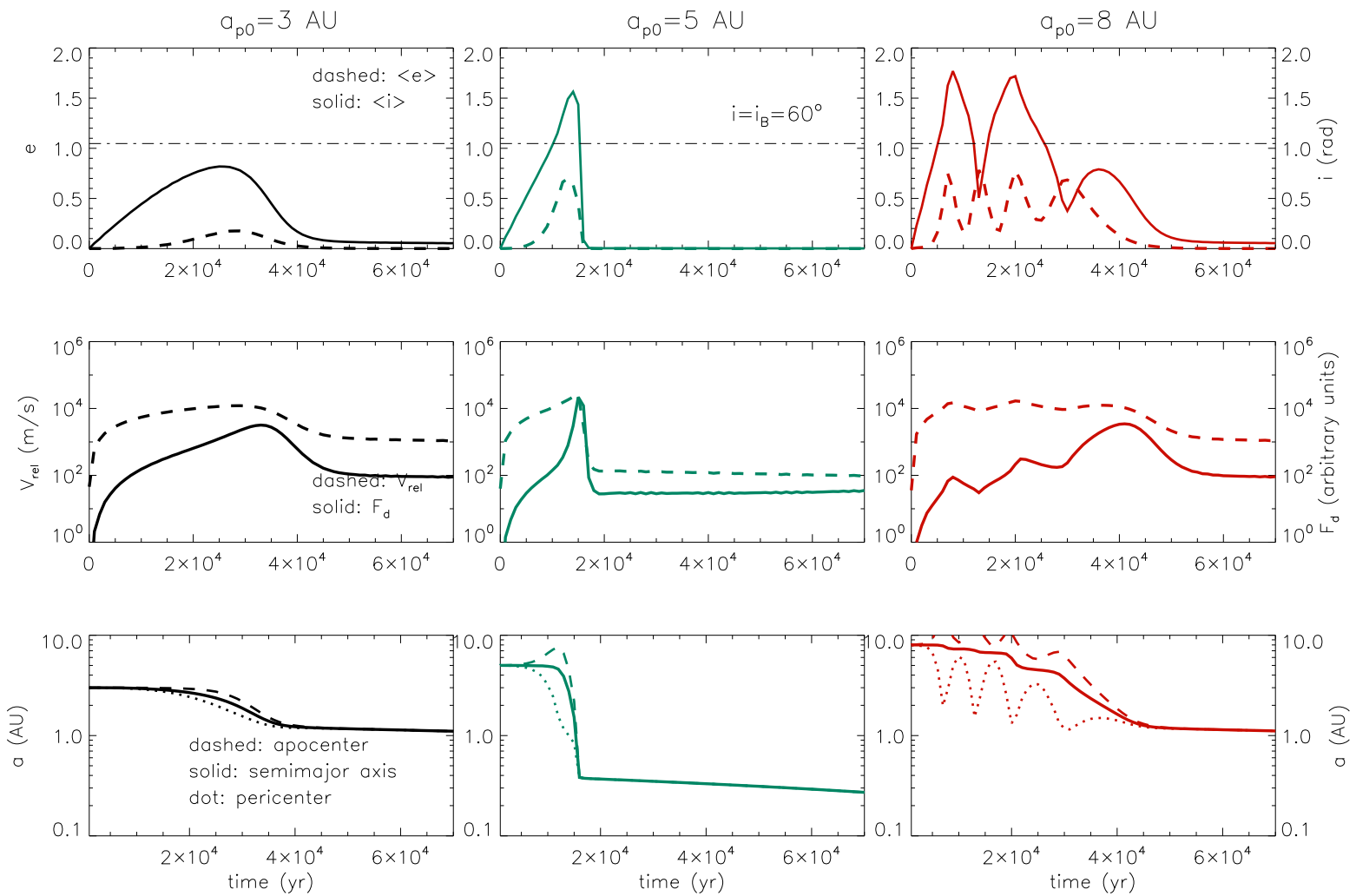

FIG. 6. - The same as Fig 5 but for the Kozai-on case with $i_{B}=60^{\circ}$. We see that high $\hat{i}$ and $\hat{e}$ (induced by the Kozai effect) significantly increase the inward migration; planetesimals can even "jump" into the very inner region, and unlike to that in the Kozai-off case, we now find that the turn-over semi-major axis (where the jumping stops) is no longer independent on $a_{p 0}$ (see Fig (7). The horizonal dot-dash line denote $i=i_{B}=60^{\circ}$ 


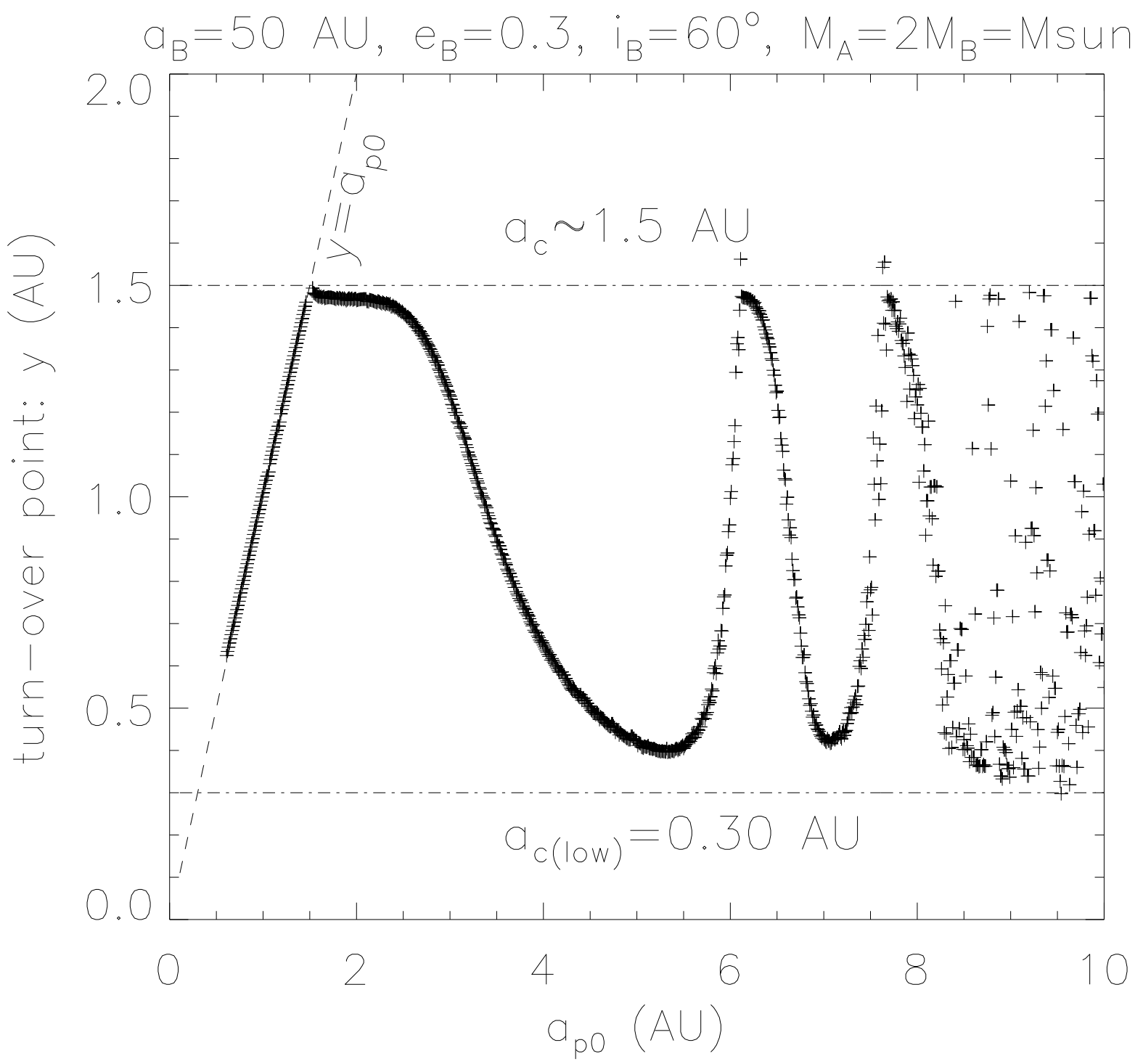

FIG. 7. - The turn-over semi-major axis for the high inclination Kozai-on case (denoted with "y" here) v.s. $a_{p 0}$ for the case with $a_{B}=50$ $\mathrm{AU}, e_{B}=0.3, i_{B}=60^{\circ}$, and $M_{A}=2 M_{B}=M_{\odot}$. Planetesimals' orbital eccentricities and inclinations are initially set as very small values $\left(<10^{-4}\right)$, and their other initial angular orbital elements are set as random values from 0 to $360^{\circ}$. We see that $y \sim a_{p 0}$ if $a_{p 0}<a_{c}$, i.e., $a_{p 0} \lesssim 1.5$ AU. For $1.5 \lesssim a_{p 0} \lesssim 8$ AU, y follows an oscillatory pattern, whose amplitude varies between a lower limit of $a_{c(l o w)} \sim 0.3$ AU and an upper limit of $a_{c}$, i.e., $y \sim 0.30-1.5 \mathrm{AU}$. For $a_{p 0}$ beyond $\sim 8 \mathrm{AU}$, the results become chaotic, but the upper and lower limits $(y \sim 0.30-1.5 \mathrm{AU})$ still hold. 


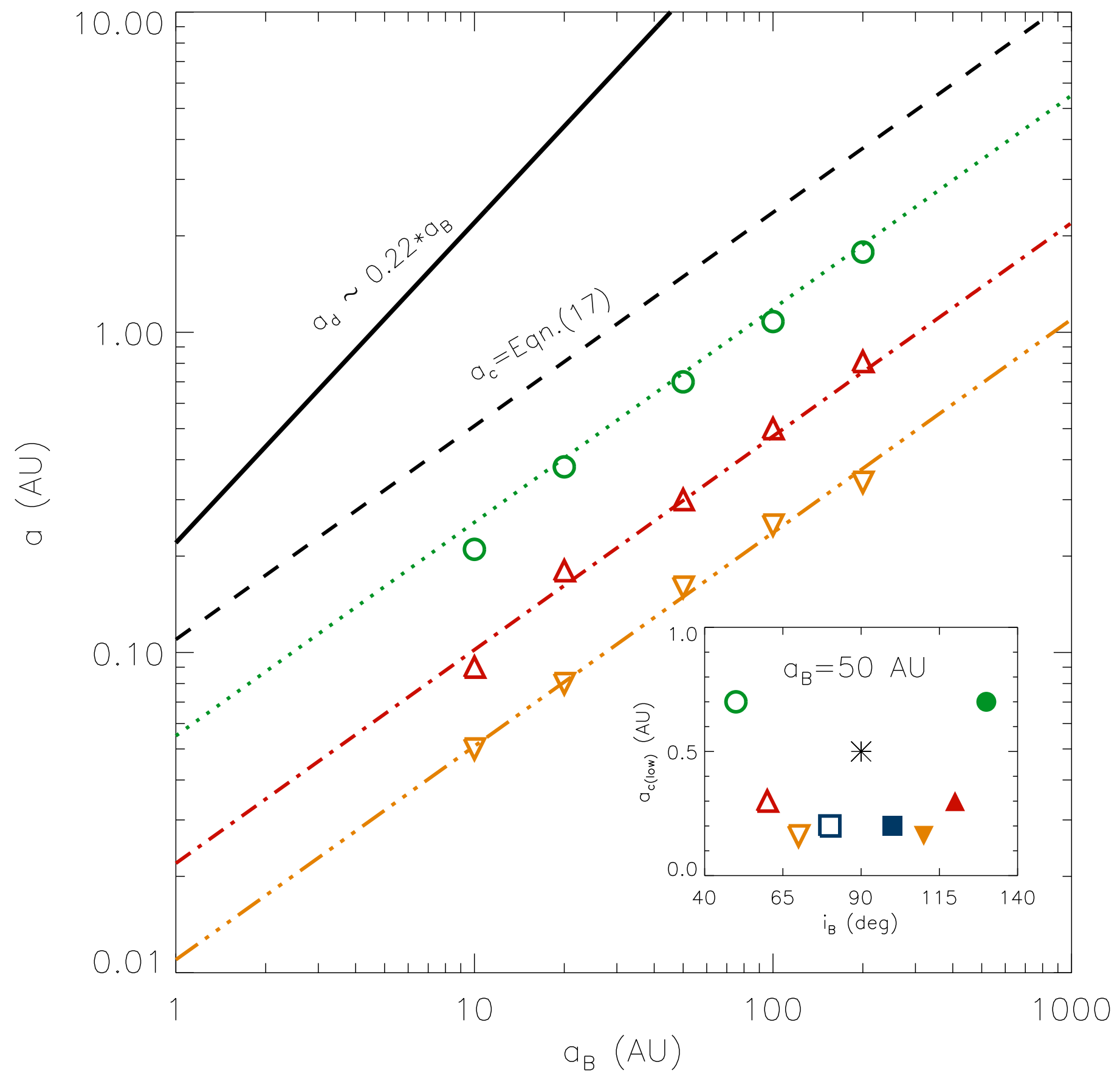

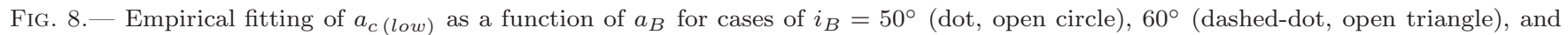
$70^{\circ}$ (dashed-dot-dot, open upside down triangle). The circle, triangle symbols are the results of individual measurements made by plotting figures similar to Fig 7 We also plot the disk stability boundary (solid line), $a_{d} \sim 0.22 a_{B}$ for $M_{A}=2 M_{B}=M_{\odot}$ and $e_{B}=0.3$ according to Holman \& Wiegert (1999), and $a_{c}$ (dashed) as references. We find that a good empirical fits for $a_{c(l o w)}$ can be given as Eq18 In the inset figure at the bottom right, we also plot the measured $a_{c(l o w)}$ as a function of $i_{B}$. The open symbols are for prograde cases while the filled ones are for the corresponding retrograde cases, while the $i=90^{\circ}$ case is plotted using an asterisk. We see that the results are symmetrical about $i=90^{\circ}$. 

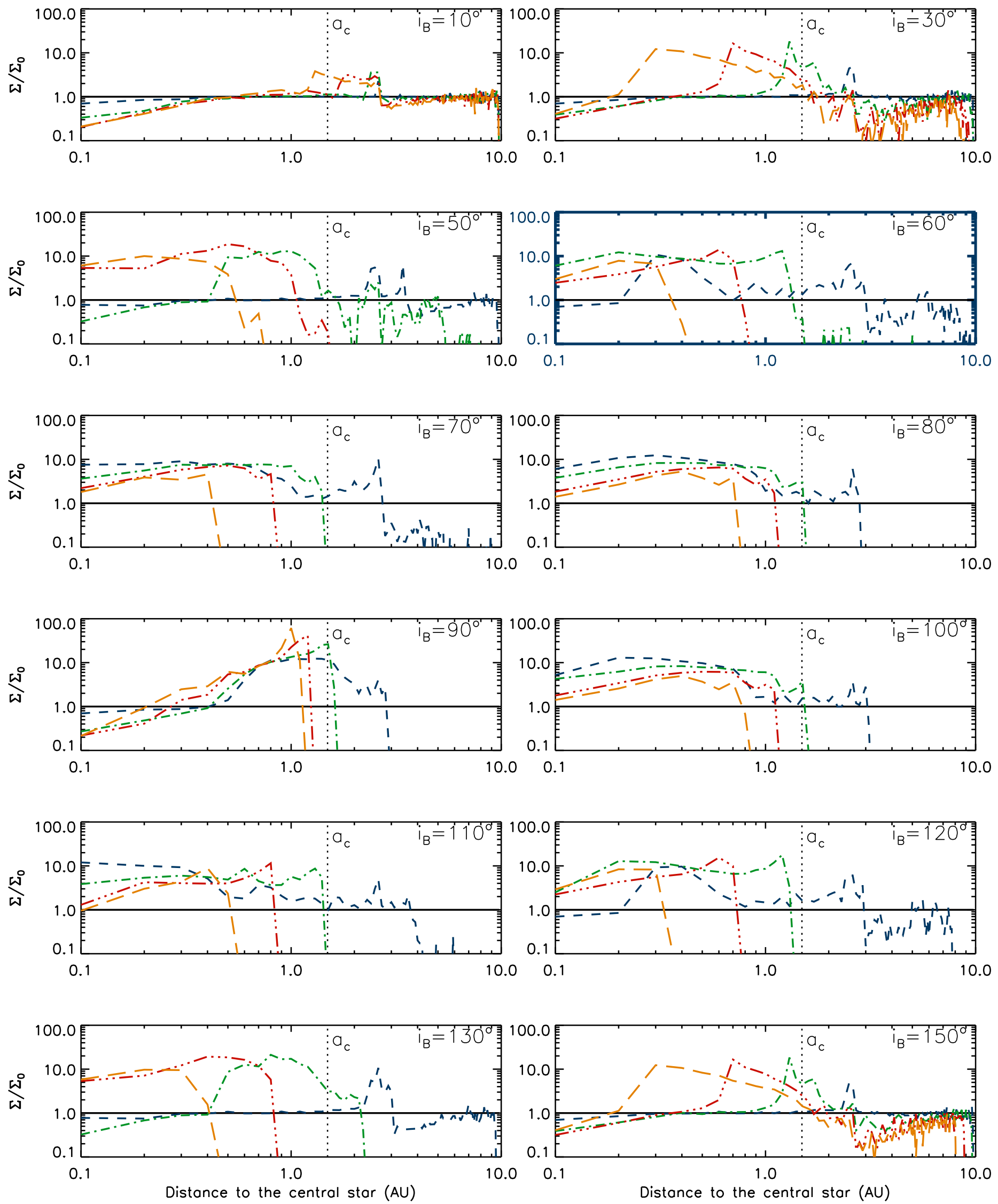

$$
\mathrm{t}=0 \mathrm{yr} \quad-\cdots \mathrm{t}=2 \times 10^{4} \mathrm{yr}-\cdots \cdots \cdot \mathrm{t}=1 \times 10^{5} \mathrm{yr}-\cdots-\cdots \mathrm{t}=3 \times 10^{5} \mathrm{yr}--\mathrm{t}=5 \times 10^{5} \mathrm{yr}
$$

FIG. 9.- Planetesimal evolution for the cases in which $M_{A}=2 M_{B}=M_{\odot}, a_{B}=50 \mathrm{AU}, e_{B}=0.3, i_{B}=10^{\circ}, 30^{\circ}, 50^{\circ}, 60^{\circ}, 70^{\circ}, 80^{\circ}$, $R_{p}=5 \mathrm{~km}$ The second panel in the right-hand column (highlighted with blue bold axes) shows the results of the standard case ( $(4.2)$. We find in highly inclined cases that the planetesimal disk surface density is highly enhanced $\left(\Sigma / \Sigma_{0} \sim 10\right)$ in the inner region within $a_{c}$, while it is significantly depleted beyond $a_{c}$. In addition, all results seems to be approximately symmetrical about $i=90^{\circ}$. For details see text in $\S 4.3 .1$ 
Xie et al.
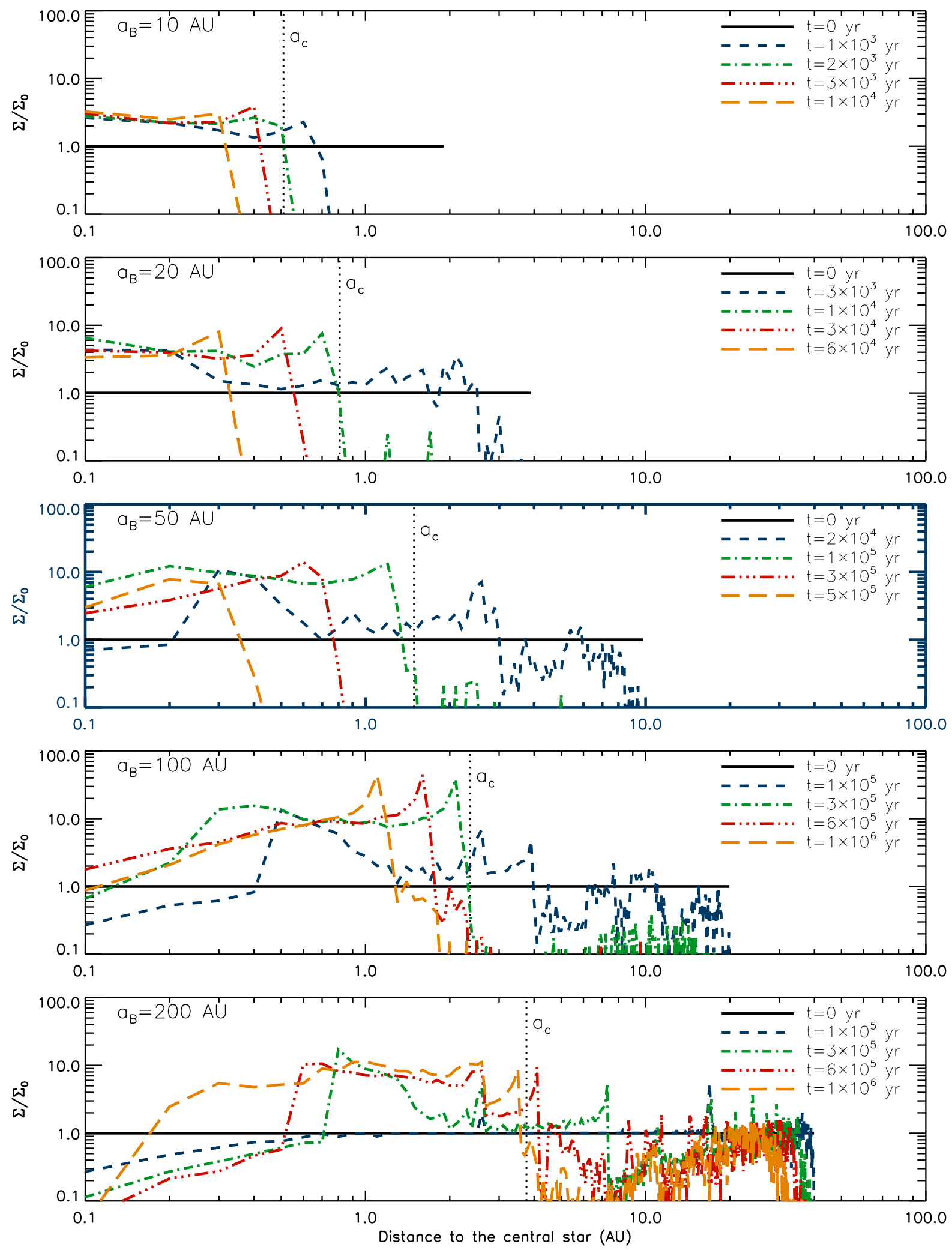

FIG. 10.- Planetesimal evolution for the cases in which $M_{A}=M_{\odot}, q_{B}=M_{B} / M_{A}=0.5, a_{B}=10,20,50,100,200,400 \mathrm{AU}, e_{B}=0.3$, $i_{B}=60^{\circ}, R_{p}=5 \mathrm{~km}$. The middle panel (highlighted with blue bold axes) shows the results of the standard case ( $(4.2)$. We see that $a_{B}$ has significant effects on determining when and to what degree the piling up effects can reach ( $\$ 4.3 .2$ ). Note, the time-sampling is different in each plot. 

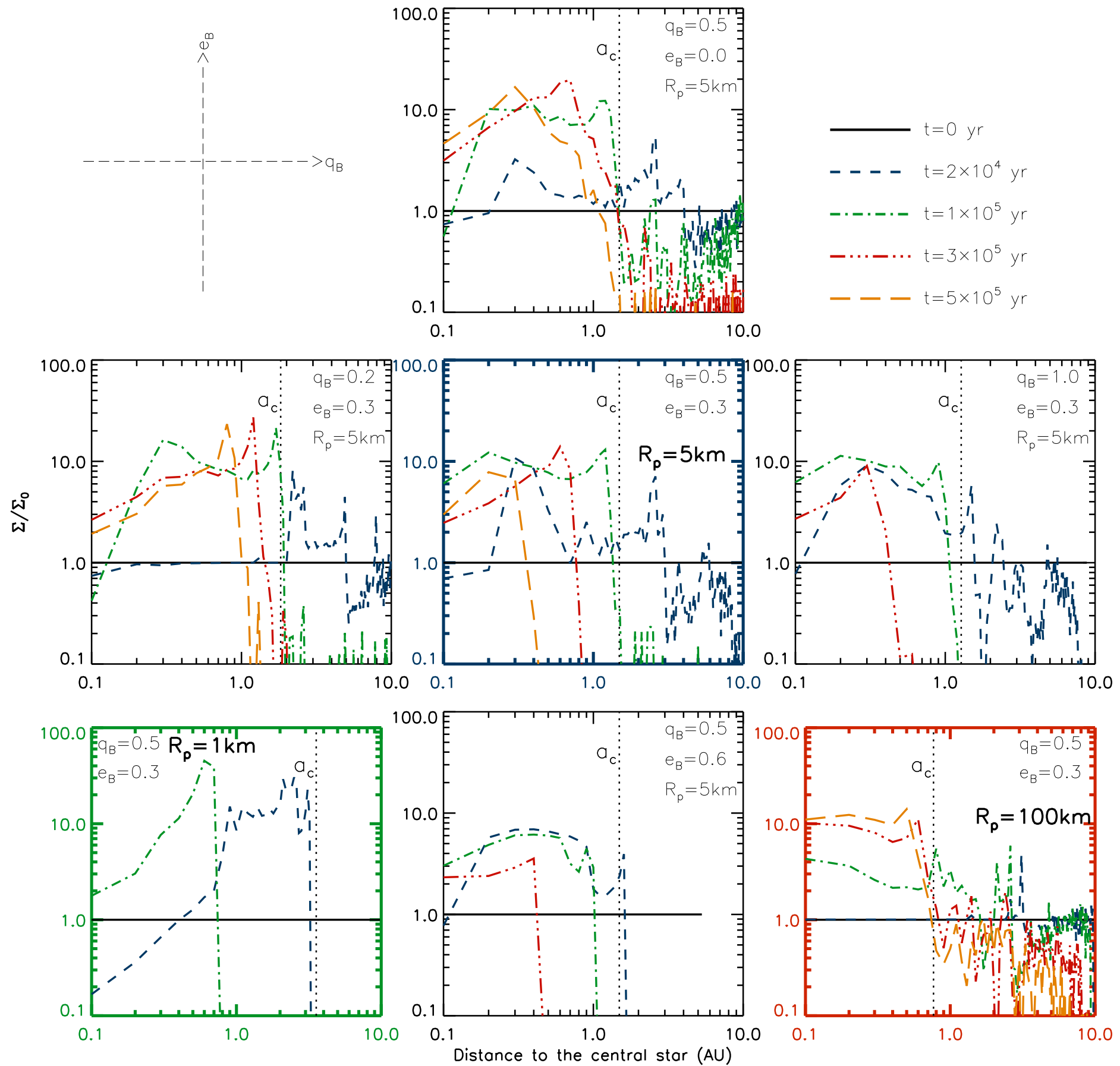

Fig. 11. - Planetesimal evolution for the cases in which $M_{A}=M_{\odot}, q_{B}=M_{B} / M_{A}=0.2,0.5,1.0, a_{B}=50$ AU, $e_{B}=0,0.3,0.6$, $i_{B}=60^{\circ}, R_{p}=5,100 \mathrm{~km}$. The middle panel (highlighted with blue bold axes) shows the results of the standard case ( $\$ 4.2$ ). The specific effects of $q_{B}, e_{B}, R_{p}$ and $f_{d}$ are summarized in $\S 4.3 .3$ In all cases, the general result holds, i.e., disk surface density is highly enhanced in the inner region within $a_{c}$, while it is significantly depleted beyond $a_{c}$. 


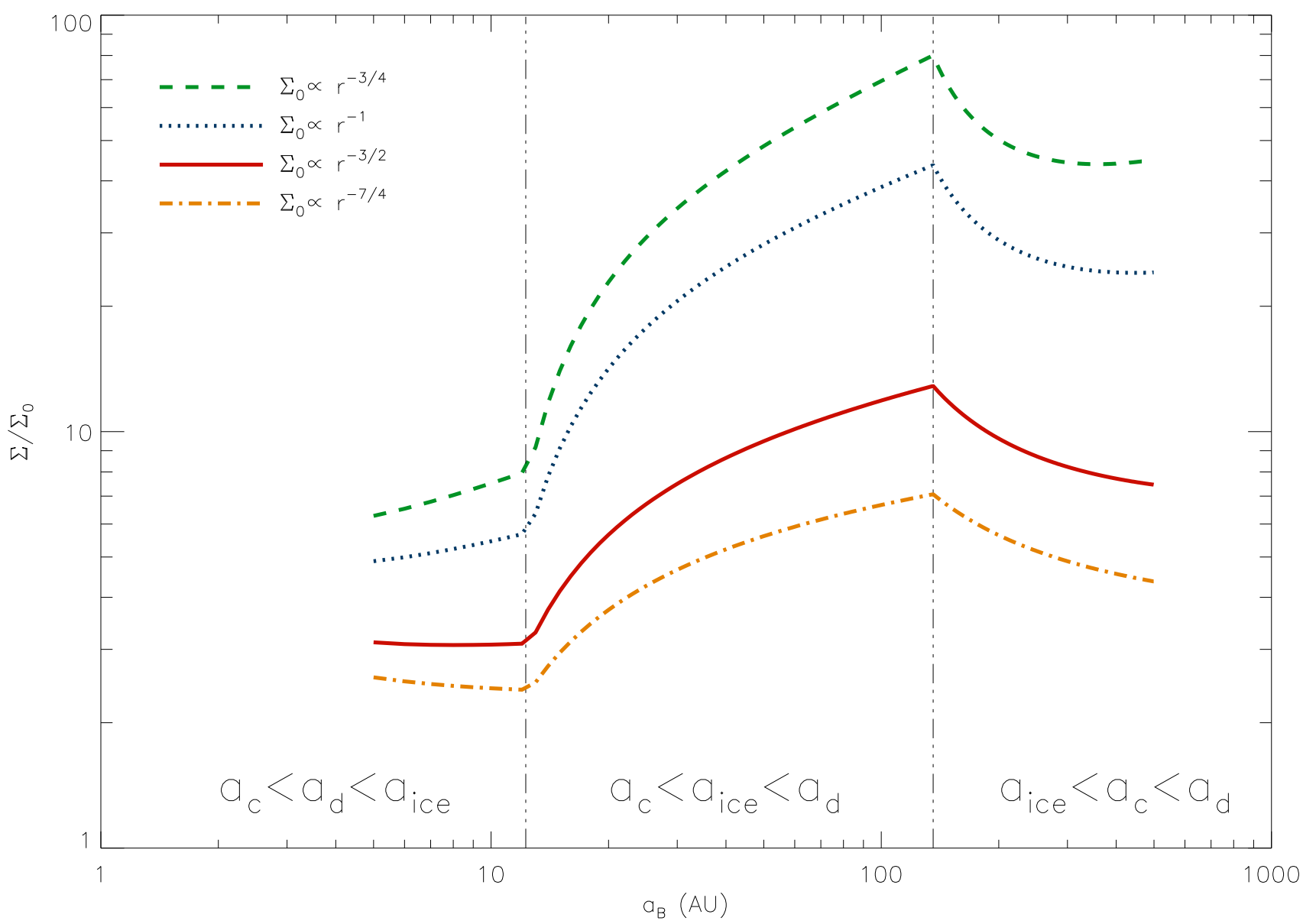

FIG. 12.- Analytical estimate (Eq21) of surface density enhancement $(\Sigma / \Sigma)$ as a function of semimajor axis of binary systems with fixed orbital eccentricity $e_{B}=0.3$ and mass ratio $M_{B} / M_{A}=0.5$. Results of four cases with different initial disk profiles are plotted in lines of different styles. The two vertical dash-dots lines, $a_{B} \sim 12.3 \mathrm{AU}$ and $a_{B} \sim 135.8 \mathrm{AU}$ mark the turn-over points where $a_{d}=a_{i c e}=2.7$ $\mathrm{AU}$ and $a_{c}=a_{i c e}=2.7 \mathrm{AU}$, respectively. 

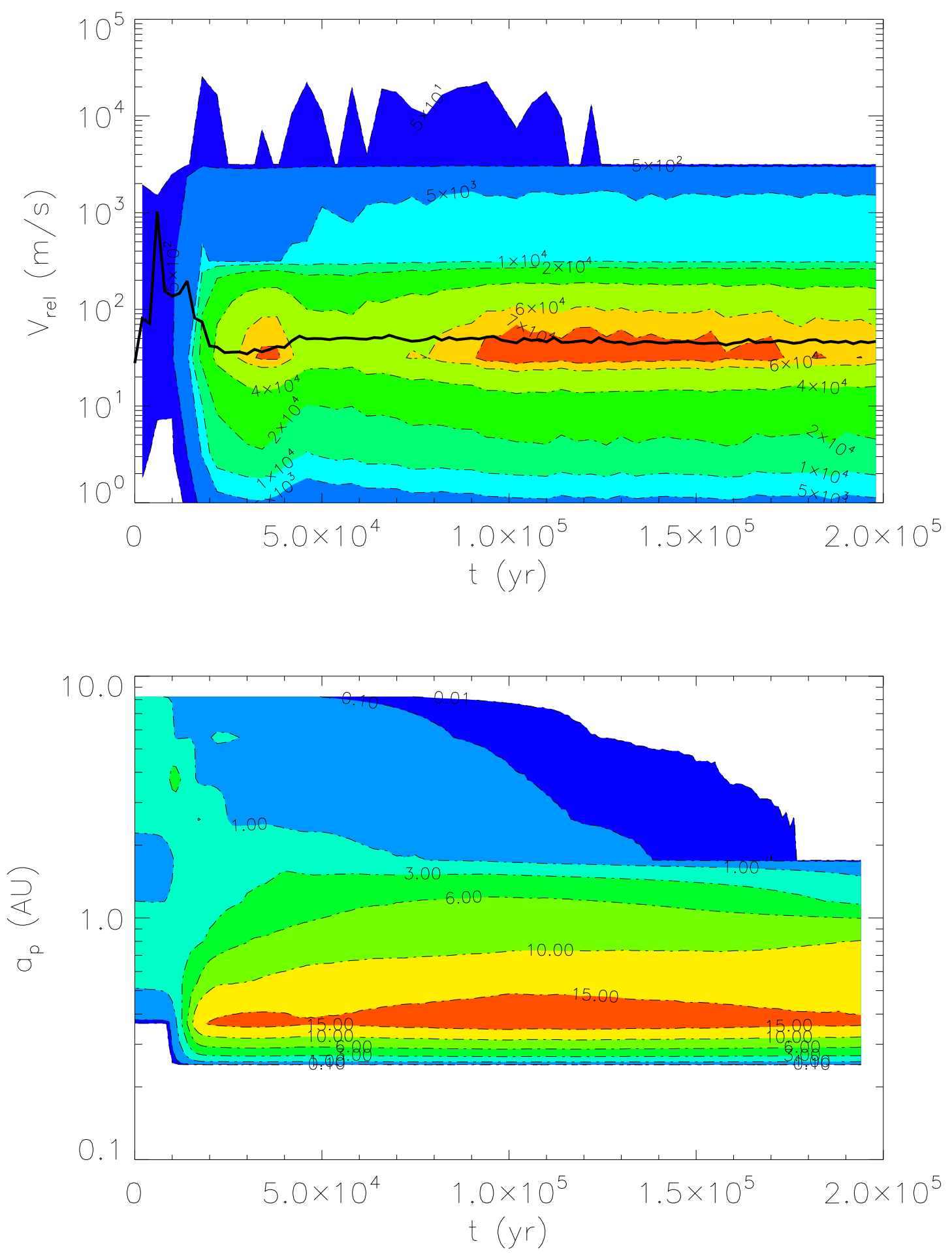

FIG. 13. - Evolution of relative velocities (top) and semi-major axis (bottom) for 10,000 planetesimals in a system with the standard binary configuration, $M_{A}=M_{\odot}, q_{B}=M_{B} / M_{A}=0.5, a_{B}=50 \mathrm{AU}, e_{B}=0.3, i_{B}=60^{\circ}$. Planetesimals with a centered Gaussian size distribution between $R_{p}=1 \mathrm{~km}$ and $R_{p}=10 \mathrm{~km}$, are initially randomly distributed between $0.3 \mathrm{AU}$ and 10 AU (flat distribution) with near circular and coplanar orbits. In the top panel, the contours denote the rate of collisions (in arbitrary units), and the thick solid line denotes the evolution of average relative velocity, for all collisions we have searched between $0.3 \mathrm{AU}$ and $10 \mathrm{AU}$. In the bottom panel, the contours denote the changes in the planetesimal surface density, i.e., $\Sigma / \Sigma_{0}$. We see that with the planetesimals piling up in the inner region, their relative velocities and collision rates are significantly reduced and increased respectively, which thus favors planetesimal growth in the inner region. 


\section{APPENDIX}

\section{MAXIMUM FORCED ECCENTRICITY}

If the secular perturbation (Kozai effect off) dominates the orbital evolution of a planetesimal. Then the average eccentricity of the planetesimal can be estimated as its forced eccentricity $e_{f}$, where

$$
e_{f} \sim \frac{5}{4} \frac{a}{a_{B}} e_{B},
$$

which is proportion to the planetesimal semimajor axis. Thus the maximum $e_{f}$ is obtained at the edge of the circumprimary disk. According to Pichardo et al. (2005), the radial size of the circumprimary disk can be estimated as

$$
R_{d} \sim R_{E g g} 0.733\left(1-e_{B}\right)^{1.20} q^{0.07}
$$

, where $R_{E g g}$ is the radius of the Roche lobe calculated by Eggleton (1983),

$$
R_{E g g}=\frac{0.49 q_{1}}{0.6 q_{1}^{2 / 3}+\ln \left(1+q_{1}^{1 / 3}\right)} a_{B} .
$$

Here, $q_{1}=M_{A} / M_{B}$ and $q 2=M_{B} /\left(M_{A}+M_{B}\right)$ are two mass ratios, and $M_{A}$ and $M_{B}$ are the masses of the primary and the secondary respectively. For a typical binary mass ratio, $q_{1}=1 / 2$, we have $R_{d} \sim 0.38 a_{B}\left(1-e_{B}\right)^{1.2}$. Substituting this $R_{d}$ into EqA1 then the maximum forced eccentricity is $e_{f} \sim 0.1$. 